CERN-TH/99-133

IFT-98/20

hep-ph/9905436

\title{
IMPLICATIONS OF THE PRECISION DATA FOR VERY LIGHT HIGGS BOSON SCENARIOS IN 2HDM(II)
}

Piotr H. Chankowski ${ }^{a, b)}$, Maria Krawczyk ${ }^{b)}$ and Jan Żochowski ${ }^{b)}$

\begin{abstract}
We present an up-to-date analysis of the constraints imposed bythe precision data on the $(C P-$ conserving) Two-Higgs-Doublet Model of type II, with emphasis on the possible existence of very light neutral (pseudo)scalar Higgs boson with mass below 20-30 GeV. We show that even in the presence of such light particles, the $2 \mathrm{HDM}(\mathrm{II})$ can describe the electroweak data with precision comparable to that given by the SM. Particularly interesting lower limits on the mass of the lighter neutral $C P$-even scalar $h^{0}$ are obtained in the scenario with a light $C P$-odd Higgs boson $A^{0}$ and large $\tan \beta$.
\end{abstract}

a) Theory Division, CERN, Geneva, Switzerland

b) Institute of Theoretical Physics, Warsaw University, Poland

CERN-TH/99-133

IFT-98/20

May 1999 


\section{Introduction}

The Standard Model (SM) of electroweak interactions is in very good agreement with the electroweak precision data collected at LEP and SLAC experiments [1]. This fact strongly supports the idea of the spontaneous breaking of the underlying $S U_{L}(2) \times U_{Y}(1)$ gauge symmetry. Yet, the actual mechanism of symmetry breaking still remains unexplored. The Higgs boson predicted by the minimal model of electroweak symmetry breaking, the SM, has not been found up to now. Only the lower limit on its mass of $\sim 90 \mathrm{GeV}$ is set by the unsuccessful search at LEP. While the extreme simplicity of the Higgs sector of the SM is theoretically appealing, there exist many of its extensions that lead to different phenomenology (more physical Higgs particles) and which also should be tested (or constrained) experimentally.

The simplest such extension is the well-known Two-Higgs-Doublet model (2HDM). It exists in several distinct versions, of which we want to consider in this article the one that is called Model II in its $C P$-conserving version (we briefly recall its structure in the next section). ${ }^{1}$ One interesting question that arises in the context of such an extension of the SM is what the available experimental limits are on the masses of the Higgs bosons predicted in such a model. In Section 3 we will recall the arguments [2] that, in the framework of the considered version of $2 \mathrm{HDM}$, the direct searches do not exclude the existence of very light neutral scalar or pseudoscalar Higgs particles. In Section 4 we show that the existence of such light Higgs bosons is notexcluded by the electroweak precision data either. An analysis of the impact of the precision data on the $2 \mathrm{HDM}(\mathrm{II})$ was already performed in the past $[6,3]$ (for the formalism and early investigations, see also [4]) but concentrated mainly on the possibility of improving the prediction for $R_{b} \equiv \Gamma\left(Z^{0} \rightarrow \bar{b} b\right) / \Gamma\left(Z^{0} \rightarrow\right.$ hadrons) (which at that time seemed to be required by the data) and on improving the $2 \mathrm{HDM}(\mathrm{II})$ global fit to the data with respect to the fit given by the SM. Since then, the experimental situation has evolved significantly. In particular the measurement of $R_{b}$ no longer shows any statistically significant deviation from the value predicted by the SM [1]. Also there are changes both in the experimental measurement and theoretical computation of the $b \rightarrow s \gamma$ decay rate, which was crucial in the analysis performed in ref. [3]. More recently a partial analysis of the constraints imposed on the 2HDM(II) by various measurements was also attempted in [5]. Here we present an up-to-date analysis of the constraints that the precision data impose on the $2 \mathrm{HDM}(\mathrm{II})$, with emphasis on the possible existence of light neutral (pseudo)scalar Higgs boson. We show that even in the presence of such light particles, the $2 \mathrm{HDM}$ (II) can describe the electroweak data with the precision comparable to that given by the SM. In this case some interesting global limits on the model can be obtained. Finally in Section 5 we summarize our results and briefly comment on the other ways the existence of light Higgs bosons are or can be constrained by other experimental data.

\footnotetext{
${ }^{1}$ The Higgs sector of the Minimal Supersymmetric Standard Model has precisely the structure of Model II, but with additional constraints imposed on the quartic couplings. However, in the following we will consider the general Model II in a regime in which it cannot be regarded as a low-energy approximation of the MSSM with heavy sparticles.
} 


\section{Two-Higgs-Doublet extension of the SM - Model II}

The multidoublet extensions of the SM are distinguished by their virtue of not introducing corrections to the $\rho$ parameter at tree level. The minimal extension of the SM consists of two doublets. The requirement of the absence at tree level of the flavour-changing neutral currents puts restrictions on how the two scalar doublets of the general 2HDM can couple to fermions. In Model II, one Higgs doublet (denoted by $\Phi_{1}$ ) couples only to leptons and downtype quarks, whereas the other doublet $\left(\Phi_{2}\right)$ couples only to up-type quarks. After spontaneous symmetry breaking, both doublets acquire vacuum expectation values $v_{1}$ and $v_{2}$, respectively with $v \equiv \sqrt{v_{1}^{2}+v_{2}^{2}}$ fixed by $M_{W}$, and

$$
\frac{v_{2}}{v_{1}} \equiv \tan \beta
$$

With two complex Higgs doublets, the 2HDM predicts the existence of five physical scalars: neutral $h^{0}, H^{0}$ and $A^{0}$ and charged $H^{ \pm}$. In the more restrictive scenario (which we are going to discuss), with $C P$ symmetry conserved by the Higgs potential, $h^{0}$ and $H^{0}$ are $C P$-even mixtures of the neutral components of the doublets (the mixing being parametrized by the angle $\alpha$ ) whereas $A^{0}$ is $C P$-odd. Thus, in the $C P$-conserving version, the Higgs sector is parametrized by four masses $M_{h}, M_{H}$ (by definition $M_{h} \leq M_{H}$ ), $M_{A}$ and $M_{H^{+}}$and three dimensionless parameters $\tan \beta, \alpha$ and $\lambda_{5}$ (for definitions see for instance, [7]). The first two dimensionless parameters are very important for the phenomenology of the Higgs sector as they determine the couplings of the physical Higgs bosons to fermions and gauge bosons: the couplings of the scalars to the down- and up-type quarks are given by the SM couplings multiplied by the factors (see for instance, $[7,8]$ )

$$
\begin{aligned}
h^{0} b \bar{b}: & -\frac{\sin \alpha}{\cos \beta}=\sin (\beta-\alpha)-\tan \beta \cos (\beta-\alpha) \\
h^{0} t \bar{t}: & \frac{\cos \alpha}{\sin \beta}=\sin (\beta-\alpha)+\cot \beta \cos (\beta-\alpha) \\
H^{0} b \bar{b}: & \frac{\cos \alpha}{\cos \beta}=\cos (\beta-\alpha)+\tan \beta \sin (\beta-\alpha) \\
H^{0} t \bar{t}: & \frac{\sin \alpha}{\sin \beta}=\cos (\beta-\alpha)-\cot \beta \sin (\beta-\alpha) .
\end{aligned}
$$

The Feynman rules for the $C P$-odd scalar couplings to fermions are given by the SM rules for $h_{S M}^{0}$ times the factors:

$$
A^{0} b \bar{b}:-i \gamma^{5} \tan \beta, \quad A^{0} t \bar{t}:-i \gamma^{5} \cot \beta
$$

Important for the direct Higgs boson search at LEP, couplings $Z^{0} Z^{0} h^{0}$ and $Z^{0} Z^{0} H^{0}$ are given by the corresponding Standard Model coupling $Z^{0} Z^{0} h_{S M}^{0}$ modified by the factors:

$$
Z^{0} Z^{0} h^{0}: \quad \sin (\beta-\alpha), \quad Z^{0} Z^{0} H^{0}: \quad \cos (\beta-\alpha),
$$

whereas the couplings of $Z^{0}$ to $A^{0} h^{0}$ and $A^{0} H^{0}$ pairs are instead proportional to

$$
Z^{0} A^{0} h^{0}: \quad \cos (\beta-\alpha), \quad Z^{0} A^{0} H^{0}: \quad \sin (\beta-\alpha) .
$$




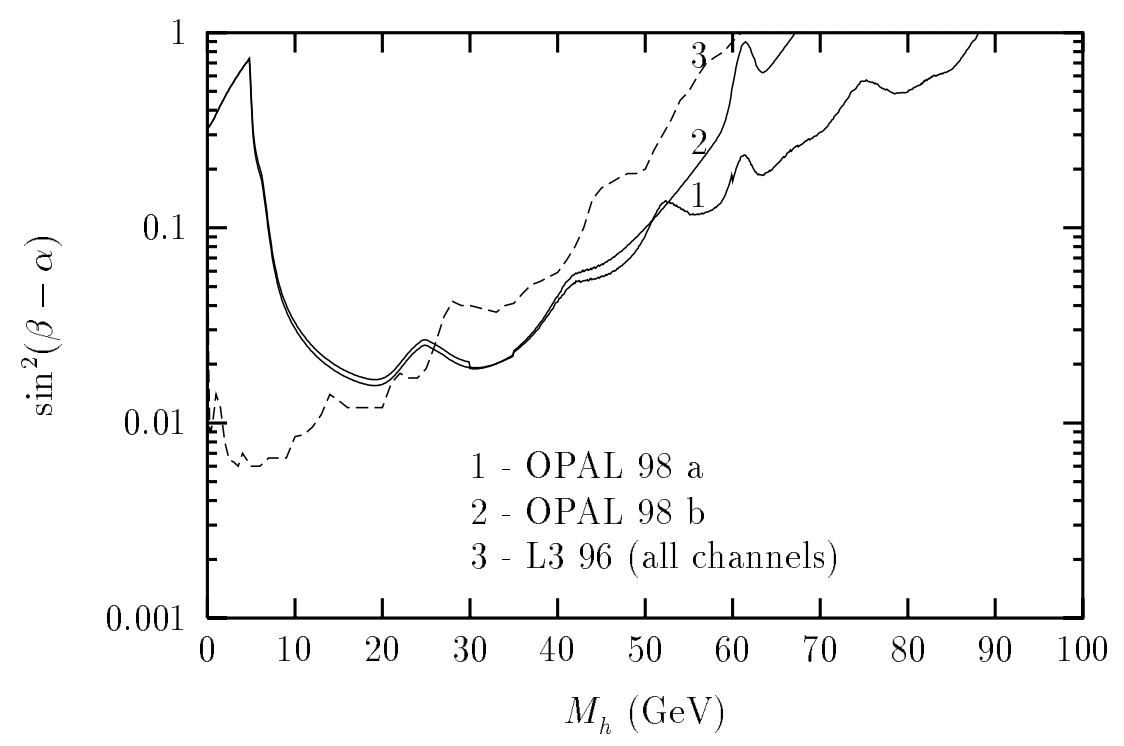

Figure 1: 95\% C.L. limits on $\sin ^{2}(\beta-\alpha)$ from the Higgs boson search at LEP as a function of $M_{h}$. Lines 1 and 2 are the OPAL results obtained assuming the Higgs boson decay branching ratios as in the SM and 100\% into hadrons, respectively [11]. Line 3 is the result of L3 [12].

It follows that, in the limit $\sin (\beta-\alpha)=1$, the lighter $C P$-even neutral Higgs boson $h^{0}$ has precisely the couplings of the Standard Model Higgs and becomes indistinguishable from it. Finally, couplings of the charged Higgs scalar to fermions, e.g. $\bar{b} t H^{-}$vertex, are given by expressions like:

$$
\frac{g}{2 \sqrt{2} M_{W}}\left[m_{t} \cot \beta\left(1+\gamma_{5}\right)+m_{b} \tan \beta\left(1-\gamma_{5}\right)\right]
$$

It is clear that for $\tan \beta$ close to zero, scalar couplings to $t \bar{t}$ pairs are strongly enhanced with respect to to the SM case, whereas for $\tan \beta$ large $(\gtrsim 10)$ the couplings to $b \bar{b}$ are enhanced. Requirement of perturbativity of both types of couplings restricts, therefore, $\tan \beta$ values to the range [9]:

$$
0.3 \lesssim \tan \beta \lesssim 130
$$

Outside this range perturbativity is lost and no firm prediction can be obtained from the model.

\section{Constraints on the 2HDM(II) from direct Higgs boson search at LEP}

Here we briefly review the constraints on the $2 \mathrm{HDM}(\mathrm{II})$ imposed by direct Higgs boson search at LEP [2], which will be taken into account in performing the fits to the electroweak data in 
Sec. 4. At the LEP collider, the Higgs boson search is based mainly on the following processes:

1. the Bjorken process $e^{+} e^{-} \rightarrow Z^{0 \star} h^{0}\left(H^{0}\right)$

2. the associated Higgs boson pair production $e^{+} e^{-} \rightarrow A^{0} h^{0}\left(H^{0}\right)$

3. the Yukawa process $e^{+} e^{-} \rightarrow b \bar{b} \rightarrow b \bar{b} A^{0}\left(h^{0}\right)$

4. $e^{+} e^{-} \rightarrow H^{+} H^{-}$.

Because of the structure of the $Z^{0} Z^{0} h^{0}$ and $Z^{0} A^{0} h^{0}$ couplings (4),(5) the processes $e^{+} e^{-} \rightarrow$ $Z^{0 \star} h^{0}$ and $e^{+} e^{-} \rightarrow A^{0} h^{0}$ are complementary to each other, provided they are simultaneously kinematically allowed. In the SM, or in the $2 \mathrm{HDM}(\mathrm{II})$ in the limit $\sin (\beta-\alpha)=1$, the nonobservation of the Bjorken process at LEP sets a lower limit on $M_{h_{S M}}$ of $\sim 90 \mathrm{GeV}$ (at $95 \%$ C.L.) [10]. In the general case, the same data put an upper limit on the factor $\sin ^{2}(\beta-\alpha)$ as a function of the lighter Higgs boson mass $M_{h}$ (see Fig. 1). The combined analysis of the complementary channels 1 and 2, performed for instance, by the OPAL collaboration [11], leads to the constraints on the $\left(M_{h}, M_{A}\right)$ plane shown in Fig. 2. It follows that the direct experimental limits on $M_{h}$ and $M_{A}$ are rather weak: the data allow for very light $h^{0}\left(A^{0}\right)$ provided $A^{0}\left(h^{0}\right)$ is heavier than $\sim 65(50) \mathrm{GeV}$ even if only $\tan \beta>1$ is allowed. In particular, there exist no absolute bound on $M_{h}$ from LEP data, provided one respects the bound on $\sin ^{2}(\beta-\alpha)$ shown in Fig. 1.

For large values of $\tan \beta$, independent constraints on $h^{0}$ and $A^{0}$ follow from the nonobservation of the Yukawa process. The available limits on the $\left(M_{A}, \tan \beta\right)$ plane are shown in Fig. 3 [15]. Similar limits on both $\left(M_{h}, \tan \beta\right)$ and $\left(M_{A}, \tan \beta\right)$ planes have been reported only recently by the DELPHI Collaboration [16].

It should also be mentioned that some, rather weak (and dependent on the assumptions made about the Higgs boson decay branching fractions) limits on the very light $h^{0}$ and $A^{0}$ can be derived from the so-called Wilczek processes [13], i.e. from $\Upsilon$ and $J / \psi$ decays into $h^{0}\left(A^{0}\right)$ and the photon [14]. We do not take these limits into account in performing the fit to the electroweak data, because the results we will show do not change as $M_{h}$ varies over the range 0-30 GeV. It should also be clear that whenever $\sin ^{2}(\beta-\alpha) \approx 0$ the heavier $C P$-even scalar, $H^{0}$, is constrained by the Bjorken process so that $M_{H} \gtrsim 90 \mathrm{GeV}$. Finally, the non-observation of the charged Higgs boson production at LEP sets the bound $M_{H^{+}}>72 \mathrm{GeV}$ [17], which is, however, much less restrictive than the indirect limit derived in the $2 \mathrm{HDM}(\mathrm{II})$ from the $b \rightarrow s \gamma$ process and, for $\tan \beta<1$, from $R_{b}$.

From the above, it follows that within the $2 \mathrm{HDM}(\mathrm{II})$ there still exist two scenarios with either very light scalar $h^{0}$ or very light pseudoscalar $A^{0}$, which are not excluded by the available data (for other constraints not discussed here, see [32, 33] and Section 5).

In the next section we will consider how the electroweak precision data constrain these two scenarios. 


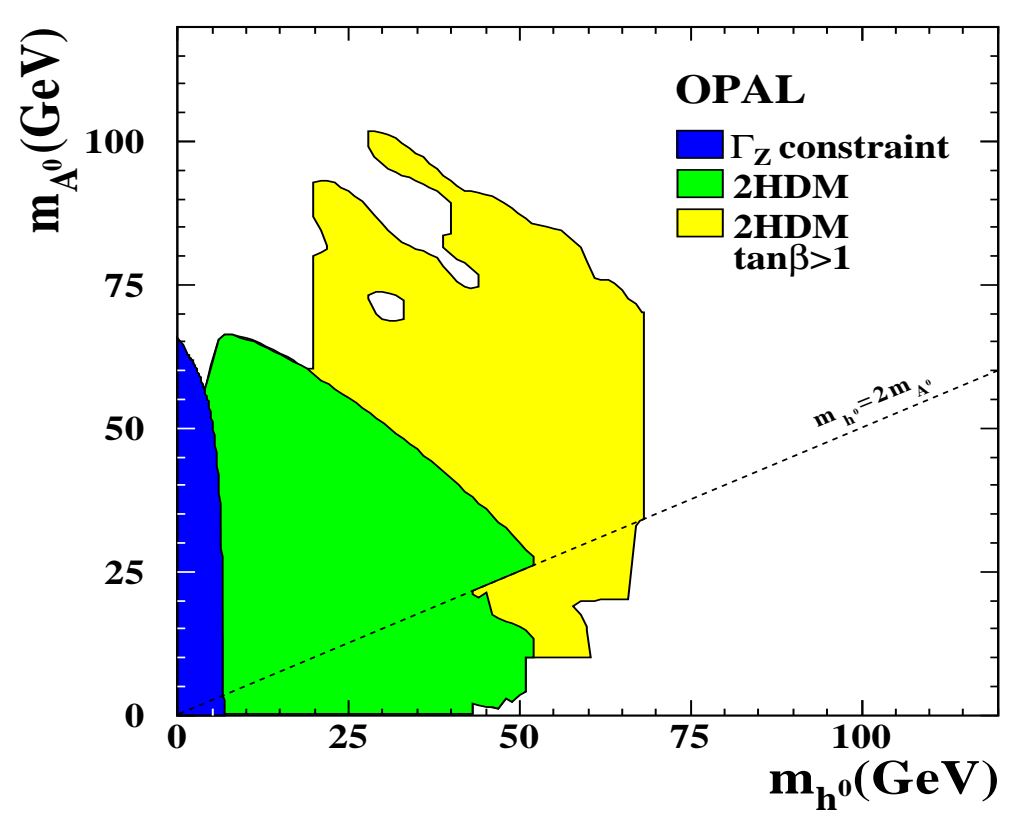

Figure 2: 95\% C.L. limits on the $\left(M_{h}, M_{A}\right)$ plane from the Bjorken process and the associated Higgs boson production as given by the OPAL collaboration [15].

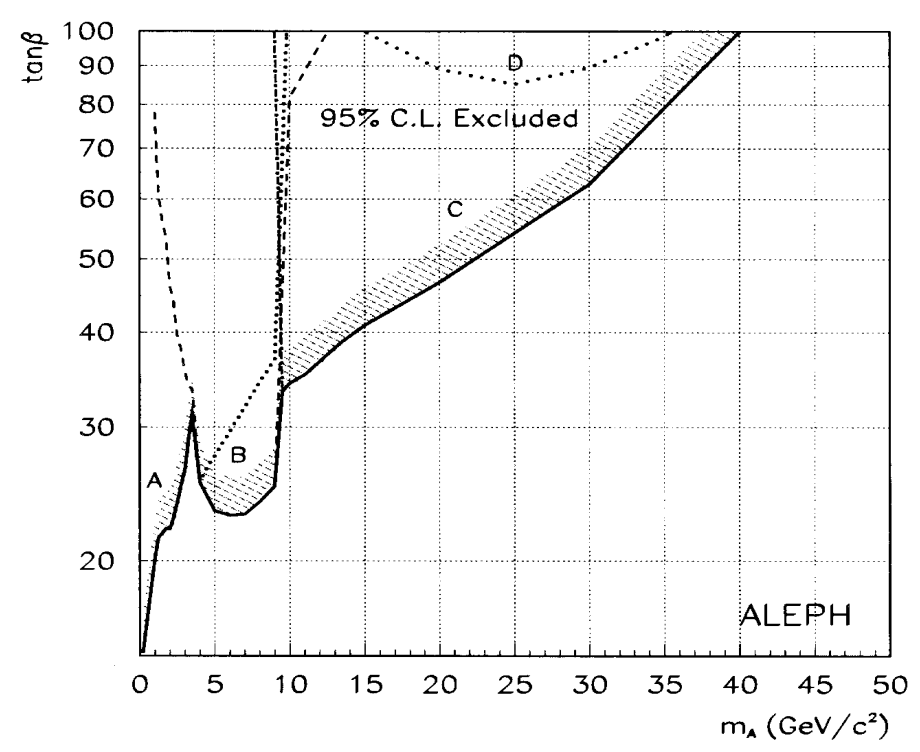

Figure 3: $95 \%$ C.L. limits on the $\left(\tan \beta, M_{A}\right)$ plane from the Yukawa process as given by the ALEPH collaboration [15]. 


\section{Global fit to the precision data}

Since the advent of LEP and SLAC experiments, precision electroweak data have been playing an increasingly important rôle in constraining the mass of the top quark in the Standard Model (SM). Nowadays, with the top quark mass directly measured at Fermilab [18], they significantly constrain the last unknown parameter of the SM, the mass of the Higgs boson [19]. They are also very useful in constraining possible forms of new physics such as supersymmetry [20] or technicolour [21]. It is, therefore, natural to ask to what extent the precision electroweak data constrain the Two Higgs Doublet Model of the type II. In this context, a question of particular importance is whether they are still compatible with the existence of a very light neutral Higgs particle (scalar or pseudoscalar one) which, as we discussed in the preceding Section, is not yet excluded by direct searches. In this Section we discuss under what conditions the existence of light $h^{0}$ or $A^{0}$ can be compatible with indirect constraints imposed by precision data.

A strictly statistical approach to constraining the $2 \mathrm{HDM}(\mathrm{II})$ indirectly would consist of finding the global minimum of the $\chi^{2}$ fit and, in the next step, excluding all points in the parameter space for which $\Delta \chi^{2}$ is greater than 3.84 (exclusion at $95 \%$ C.L.). Comparison of such an analysis with the similar one carried for the SM would reveal that, per degree of freedom (d.o.f.), the fit in $2 \mathrm{HDM}$ (II) is much worse than in the SM. This follows from the fact that the description of the electroweak data by the latter is nearly perfect [1]. Hence, a $2 \mathrm{HDM}(\mathrm{II})$ predicting individual observables as accurately as the SM would have much worse $\chi^{2} /$ d.o.f., because of larger number of free parameters in its Higgs potential. In our investigation we do not follow such an approach. Rather, we take the SM best global $\chi^{2}$ value as a reference point and concentrate on the qualitative discussion of which regions of the $2 \mathrm{HDM}(\mathrm{II})$ parameter space can give an equally good global $\chi^{2}$ value. In order not to be too restrictive, when constraining the parameter space we use the rough criterion that the $\chi^{2}$ in the $2 \mathrm{HDM}(\mathrm{II})$ should not be greater than the SM best $\chi^{2}$ value plus 4 . Our emphasis is, however, on the fact that such a criterion (in fact any reasonable one) does allow for a very light neutral scalar or pseudoscalar. The data we take into account include the precision electroweak data reported at the Moriond '98 conference [1], which are dominated by those from LEP 1 . For future reference we record that the best SM fit to the electroweak data we have chosen gives us $\chi^{2} \approx 15.5$. Therefore, all bounds on the masses of the 2HDM(II) Higgs bosons we will present are derived by requiring the $\chi^{2}$ in that model to be less than 19.5.

In discussing the values taken by the $\chi^{2}$ for various Higgs boson mass configurations (to explain qualitatively the origins of the bounds we show) we will always start with a discussion of the contribution to the $\Delta \rho$ parameter, defined as

$$
\Delta \rho=\frac{\Pi_{W W}(0)}{M_{W}^{2}}-\frac{\Pi_{Z Z}(0)}{M_{Z}^{2}}-2 \frac{s_{W}}{c_{W}} \frac{\Pi_{Z \gamma}(0)}{M_{Z}^{2}},
$$

where $s_{W}\left(c_{W}\right)$ is the sine (cosine) of the Weinberg angle. It largely determines the bulk of the predicted values of the electroweak observables such as $M_{W}, \sin ^{2} \theta^{e f f}$ (measured through various asymmetries of the final fermions and/or their polarizations [1]), etc., and is therefore the main factor shaping the $\chi^{2}$ curves, at least for not too small or too large values of $\tan \beta$, i.e. when the couplings of the Higgs bosons to the $b \bar{b}$ pair are not enhanced. 
In addition, for low and high values of $\tan \beta$, a particularly important rôle is played in the fit by the quantity $R_{b}$. The current experimental result is $R_{b}=0.21656$, with error $\Delta R_{b}=0.00074$ (which is 0.9 standard deviation above the SM prediction). Its importance follows from the fact that, in the $2 \mathrm{HDM}(\mathrm{II})$, the contribution of the Higgs bosons can easily change the prediction for $R_{b}$ with respect to the SM, spoiling the $\chi^{2}$ fit to the data. This contribution has been studied in detail in ref. [22]. (Handy formulae for $\delta R_{b}$ can also be found in the Appendix of ref. [23].) The contribution of $H^{+}$to $\delta R_{b}$ contains parts that are proportional to $\left(m_{t} / M_{Z}\right)^{2} \cot ^{2} \beta$ and $\left(m_{b} / M_{Z}\right)^{2} \tan ^{2} \beta$ and, consequently, can be sizeable for either very small or very large values of $\tan \beta$. The neutral scalars become relevant to $R_{b}$ only in the latter limit (see. Eqs. (2) and (3)). For a qualitative understanding of the results it is sufficient to remember that the contribution of $\mathrm{H}^{+}$is always negative, whereas the contribution of the neutral Higgs bosons can be positive provided $A^{0}$ is not too heavy, say $M_{A} \lesssim 100 \mathrm{GeV}$, and the splitting between its mass and the mass of the $C P$-even scalar $h^{0}$ or $H^{0}$ (the one which, for a given angle $\alpha$, couples more strongly to the $b \bar{b}$ pair) is not too large. For example, in the configuration (typical for the Minimal SUSY Standard Model with large $\tan \beta$ ) $M_{h} \sim M_{A} \lesssim 70 \mathrm{GeV}$ (and $\sin ^{2} \alpha \approx 1$ ) the contribution of $A^{0}$ and $h^{0}$ can easily overcompensate the negative contribution of $H^{+}$with mass $M_{H^{+}}>100 \mathrm{GeV}$. It is the interplay of the contribution to the $\Delta \rho$ parameter and the contribution to $\delta R_{b}$ that is responsible for interesting bounds on $2 \mathrm{HDM}(\mathrm{II})$ with light (pseudo)scalar particle, which can be derived on the basis of the $\chi^{2}$ fit.

Another very important constraint on new physics is the measured value of the branching ratio $B R\left(B \rightarrow X_{s} \gamma\right)$ [24]. In the context of the $2 \mathrm{HDM}(\mathrm{II})$ this measurement can be converted into a lower bound on the mass of the charged Higgs boson [25]. Recently a big effort was made by various groups to improve the accuracy of the theoretical prediction for this ratio [26, 27, 28]. Our lower bound on $M_{H^{+}}$, based on $b \rightarrow s \gamma$ for $m_{t}=174 \mathrm{GeV}$, is shown as a function of $\tan \beta$ in Fig. 6 a by the solid line. ${ }^{2}$ It is (for large $\tan \beta$ ) higher by some $35 \mathrm{GeV}$ than the recent estimate [30], which gives $M_{H^{ \pm}} \gtrsim 165 \mathrm{GeV}$. In view of the well-known exquisite sensitivity of the limit on $M_{H^{+}}$to the details of the analysis, this should be considered as a satisfactory agreement. We will, however, try to keep open the possibility that the charged Higgs boson can be as light as $\sim 165 \mathrm{GeV}$.

\subsection{Light $h^{0}$}

We discuss the light $h^{0}$ scenario first. For the sake of clarity it is convenient to distinguish two $h^{0}$ mass ranges: i) $M_{h} \lesssim 30 \mathrm{GeV}$ and ii) $M_{h}>30 \mathrm{GeV}$. This division follows from the upper bounds imposed by the direct LEP search on the allowed value of $\sin ^{2}(\beta-\alpha)[12]$ (Fig. 1): in the case $i$ ) $\sin ^{2}(\beta-\alpha)<0.01-0.02$ (and for practical purposes can be set to zero); in the case $i i$ ) the

\footnotetext{
${ }^{2}$ We compute $B R(b \rightarrow s \gamma)$ with NLO accuracy, following the approach of ref. [29] supplemented with electromagnetic as well as $1 / m_{b}^{2}$ and $1 / m_{c}^{2}$ corrections setting the parameter $\delta=0.9$ [28]. The theoretical uncertainty is taken into account by computing the rate for $\mu_{b}=2.4$ and $9.6 \mathrm{GeV}$ and then shifting its larger (smaller) value upward (downward) by the errors, added in quadrature, related to the uncertainties in $\alpha_{s}, m_{b}$, $m_{c} / m_{b},\left|V_{t b} V_{t s}^{\star} / V_{c b}\right|^{2}$, and higher-order electroweak corrections; we do not take into account the variation of the scale $\mu_{W}$. If the resulting band of theoretical predictions for $B R(b \rightarrow s \gamma)$ has an overlap with the CLEO $95 \%$ C.L. band, the point is allowed.
} 

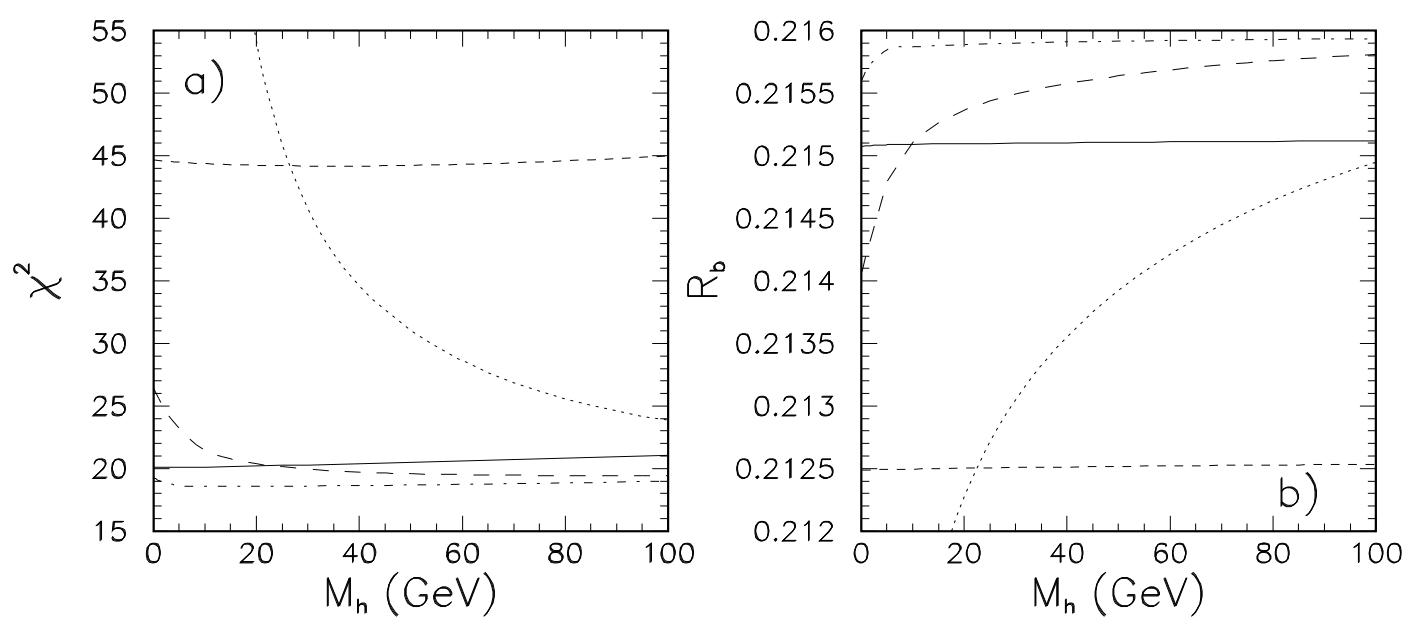

Figure 4: $\chi^{2}$ and $R_{b}$ as a function of $M_{h}$ in the case of triple degeneracy with $M_{D} \equiv M_{H}=$ $M_{A}=M_{H^{+}}=250 \mathrm{GeV}$ and $\sin ^{2}(\beta-\alpha)=0$. Short-dashed, solid, dot-dashed, long-dashed and dotted lines correspond to $\tan \beta=0.5,1,5,20$ and 50, respectively. The top mass is fixed to $m_{t}=174 \mathrm{GeV}$.

upper bound on $\sin ^{2}(\beta-\alpha)$ changes roughly linearly (on the logarithmic scale) from $\sim 0.02$ for $M_{h} \approx 30 \mathrm{GeV}$ up to 1 for $M_{h} \approx 90 \mathrm{GeV}$ (see Fig. 1 ). In this paper we will be mainly interested in case $i$ ) and will essentially not explore the case $i i$ ), which requires a more involved analysis. It will also prove helpful to consider separately two ranges of the parameter $\tan \beta \equiv v_{2} / v_{1}$, namely $0.5 \lesssim \tan \beta \lesssim 10-20$ (small and intermediate) and $20 \lesssim \tan \beta \lesssim 50$ (large). Distinct properties of these regions follow from the different sensitivity of the important observable $R_{b}$ to the masses of the Higgs bosons for small and intermediate values of $\tan \beta$, and for large ones.

In order to understand the main features of the $\chi^{2}$ fit to the electroweak data qualitatively, it is instructive to begin the discussion of the light $h^{0}$ case with the somewhat peculiar limit in which the remaining Higgs bosons, $H^{ \pm}, A^{0}$ and $H^{0}$, are exactly degenerate and have a common mass $M_{D}$. In Fig. 4a we show the value of $\chi^{2}$ obtained in the $2 \mathrm{HDM}(\mathrm{II})$ as a function of $M_{h}$ for different values of $\tan \beta$ and $M_{D}=250 \mathrm{GeV}$. Corresponding values of $R_{b}$ are shown in Fig. $4 \mathrm{~b}$. In both cases we set $\sin ^{2}(\beta-\alpha)=0$ and we keep $m_{t}$ fixed at $174 \mathrm{GeV}$. The pattern observed in Fig. 4a can be easily understood by checking the contributions to the parameter $\Delta \rho$, which can be represented, in the $2 \mathrm{HDM}[7]$, in the convenient form:

$$
\Delta \rho=\frac{\alpha}{4 \pi s_{W}^{2} M_{W}^{2}} A\left(M_{A}, M_{H^{+}}\right)+\cos ^{2}(\beta-\alpha) \Delta_{c}+\sin ^{2}(\beta-\alpha) \Delta_{s},
$$

where

$$
\Delta_{c}=\frac{\alpha}{4 \pi s_{W}^{2} M_{W}^{2}}\left[A\left(M_{H^{+}}, M_{h}\right)-A\left(M_{A}, M_{h}\right)\right]+\Delta \rho_{S M}\left(M_{H}\right)
$$



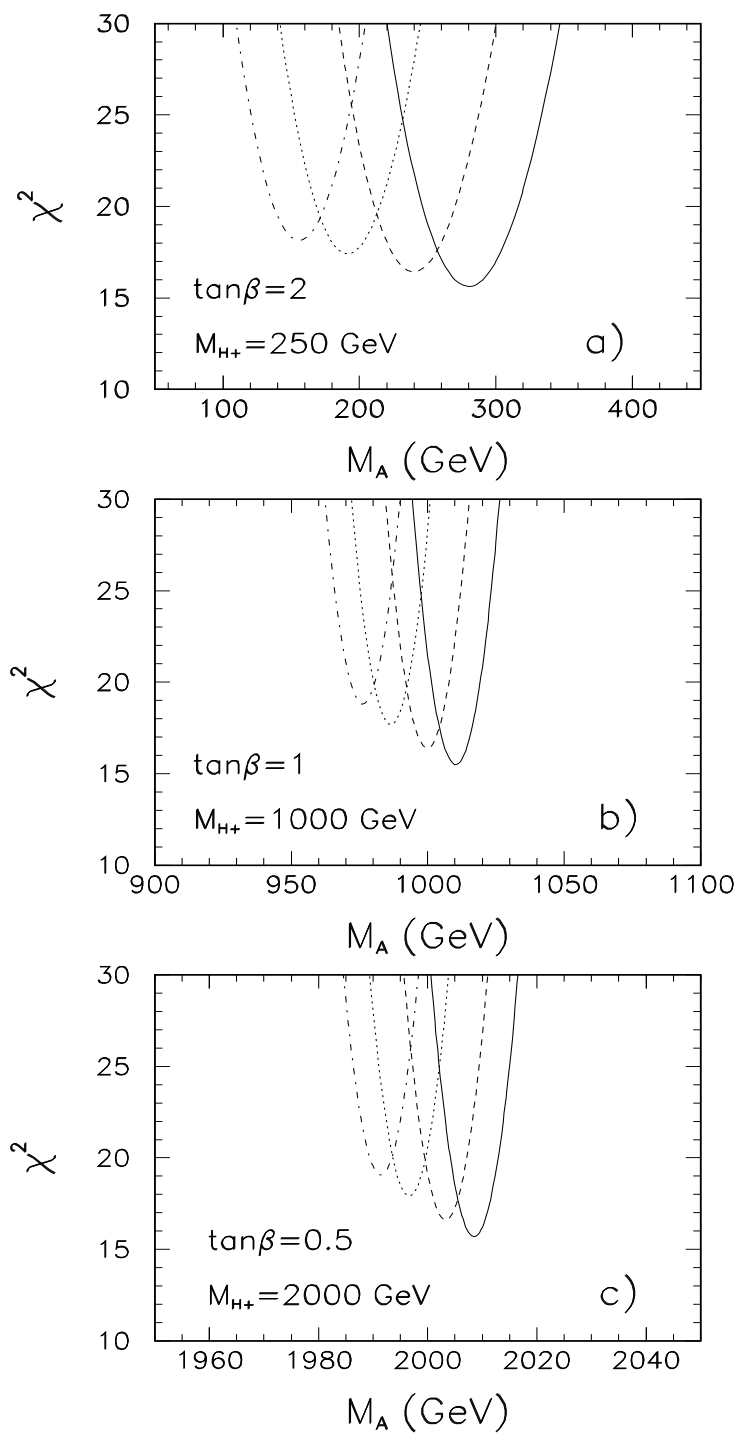

Figure 5: $\chi^{2}$ for $M_{h}=20 \mathrm{GeV}$ and $\sin ^{2}(\beta-\alpha)=0$ as a function of $M_{A}$ for different low and intermediate values of $\tan \beta$ and different $H^{+}$masses. Solid, dashed, dotted and dot-dashed lines correspond to $M_{H}=90,200,500$ and $1000 \mathrm{GeV}$, respectively; $m_{t}=174 \mathrm{GeV}$. 
and $\Delta_{s}$ is obtained by the exchange $M_{h} \leftrightarrow M_{H}$. The function

$$
A(x, y)=A(y, x) \equiv \frac{1}{8} x^{2}+\frac{1}{8} y^{2}-\frac{1}{4} \frac{x^{2} y^{2}}{x^{2}-y^{2}} \log \frac{x^{2}}{y^{2}}
$$

is positive and large if $x \gg y$ and vanishes for $x=y$. Finally,

$$
\begin{aligned}
\Delta \rho_{S M}(M) & =\frac{\alpha}{4 \pi s_{W}^{2} M_{W}^{2}}\left[A\left(M, M_{W}\right)-A\left(M, M_{Z}\right)\right] \\
& +\frac{\alpha}{4 \pi s_{W}^{2}}\left[\frac{M^{2}}{M^{2}-M_{W}^{2}} \log \frac{M^{2}}{M_{W}^{2}}-\frac{1}{c_{W}^{2}} \frac{M^{2}}{M^{2}-M_{Z}^{2}} \log \frac{M^{2}}{M_{Z}^{2}}\right]
\end{aligned}
$$

is the Standard Model Higgs boson contribution to $\Delta \rho$. A good quality of the fit is obtained for

$$
\Delta \rho_{N E W}=\Delta \rho-\Delta \rho_{S M}\left(M_{h_{S M}^{0}}\right) \approx 0
$$

where $M_{h_{S M}^{0}}$ is a reference SM Higgs boson mass $\approx 100 \mathrm{GeV}$.

Since in the case of triple degeneracy shown in Fig. $4 \Delta \rho$ is independent of $\tan \beta, \sin ^{2}(\beta-\alpha)$ and $M_{h}$ values, for fixed $M_{D}$ the $\chi^{2}$ curves reflect mainly ${ }^{3}$ the dependence of $R_{b}$ on $M_{h}$ for different values of $\tan \beta$. For low and moderate values of $\tan \beta$ the predicted value of $R_{b}$ is $M_{h}$-independent, whereas for large $\tan \beta(\gtrsim 20)$, for which the $h^{0} b \bar{b}$ coupling is enhanced (see Eq. (2)), the sensitivity of $R_{b}$ to $M_{h}$ becomes crucial. In addition, for light $H^{+}$(i.e. small $M_{D}$, $\sim 200 \mathrm{GeV}$ ) its negative contribution to $R_{b}$ spoils the $\chi^{2}$ fit for very small or very large values of $\tan \beta$ (but this effect is $M_{h}$-independent). On top of that comes the global sensitivity of the fit to $M_{D}$, which enters through $\Delta \rho_{S M}\left(M_{D}\right)$. This dependence is exactly the same as the dependence of the SM fit (for fixed top quark mass) on the value of the SM Higgs boson mass. It follows that the best fit (in the case of triple degeneracy) is obtained for $M_{D}$ as low as possible and intermediate values of $\tan \beta$. Note that only the case of $M_{D}=250 \mathrm{GeV}$ and $\tan \beta=5$ (and marginally, for heavier $h^{0}$, also for $\tan \beta=20$ ) shown in Fig. 4 yields an acceptable value of $\chi^{2}$. Allowing for $M_{D}=165 \mathrm{GeV}$ gives (for intermediate values of $\tan \beta$ ) $\chi^{2} \approx 17.5$. In the region i) taking $\sin ^{2}(\beta-\alpha)=0.01$ improves $\chi^{2}$ only marginally (it decreases by $\sim 0.2$ for $M_{D}=250$ $\mathrm{GeV})$. In the region ii) taking the largest value of $\sin ^{2}(\beta-\alpha)$ allowed by the LEP data, e.g. $\sin ^{2}(\beta-\alpha)=0.2$ for $M_{h}=50, \mathrm{GeV}$ decreases for $M_{D}=250 \mathrm{GeV}$ the $\chi^{2}$ value to $\sim 18.1$ as a result of a "redistribution" of contributions in the last two terms in Eq. (8).

Essential improvement of the fit is, however, obtained by the departure from the strict limit of triple degeneracy. For very small and intermediate values of $\tan \beta$, this is illustrated in Fig. 5 where for $M_{h}=20 \mathrm{GeV}$ (which is representative of $0<M_{h} \lesssim 30 \mathrm{GeV}$ ), $\sin ^{2}(\beta-\alpha)=0$, and $m_{t}$ fixed at $174 \mathrm{GeV}$, we show $\chi^{2}$ as a function of $M_{A}$ for different combinations of $H^{+}$and $H^{0}$ masses.

To explain the pattern of $\chi^{2}$ seen in Figs. 5a for $\tan \beta=2$ (which is representative of intermediate values of $\tan \beta$ for which the predicted value of $R_{b}$ is as in the SM), recall that

\footnotetext{
${ }^{3}$ For a qualitative explanation of the shapes of the $\chi^{2}$ curves the other "oblique" parameters, $S$ and $U$ [31], play only a secondary rôle.
} 

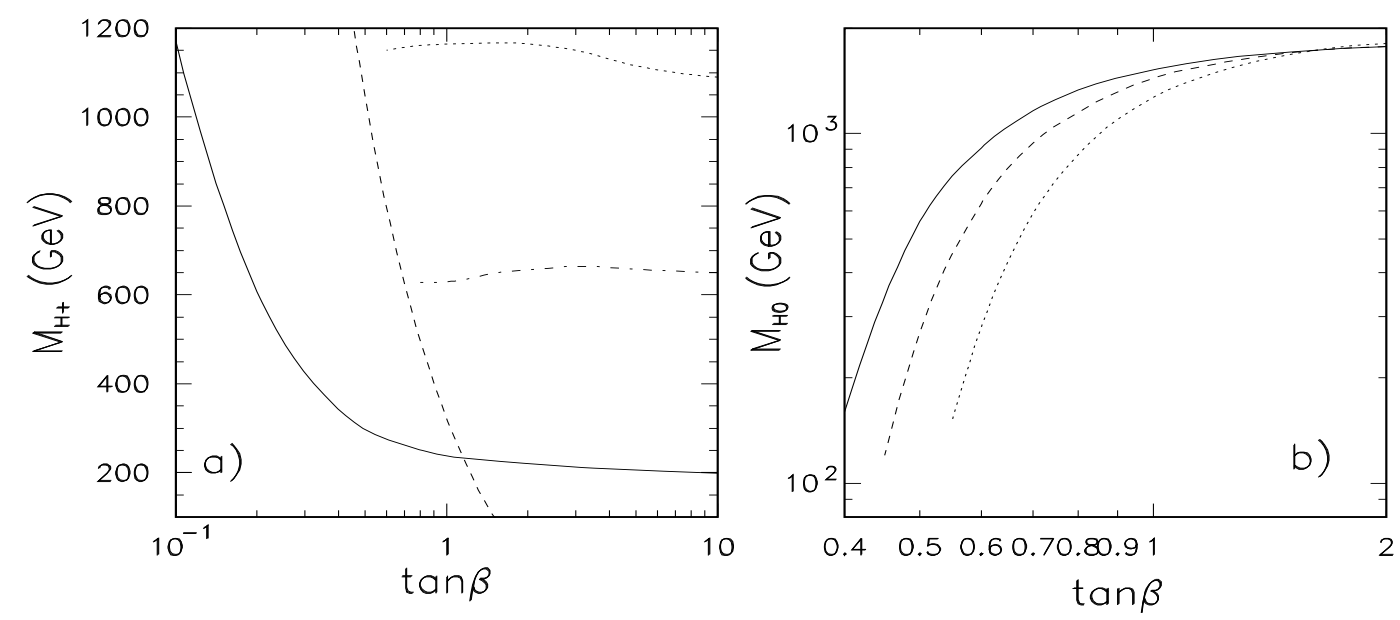

Figure 6: a) Lower limits on $H^{+}$mass coming separately from $b \rightarrow s \gamma$ (solid line) and from the requirement that $R_{b}^{2 H D M}>R_{b}^{E X P}-2\left(\Delta R_{b}\right)^{E X P}$ (dashed line) as a function of $\tan \beta$. Also shown are upper limits on $M_{H^{+}}$arising for $M_{h}<20 \mathrm{GeV}$ from the requirement of fine tuning in $M_{A}$ not larger than $1 \%$ and $3 \%$ (dotted and dash-dotted lines, respectively). b) Upper limits on $M_{H}$ in the case of light $h^{0}\left(M_{h} \leq 20-30 \mathrm{GeV} ; \sin (\beta-\alpha)=0\right)$ and $M_{H^{+}}=1000,800$ and $600 \mathrm{GeV}$ (solid, dashed and dotted lines, respectively).

the SM-type contribution (11) to $\Delta \rho$, Eq. (8), is negative and (for $\sin ^{2}(\beta-\alpha)=0$ ) decreases with increasing $H^{0}$ mass. This too negative contribution can be easily compensated for by the contribution of the other Higgs bosons to $\Delta \rho$, when the equality of $H^{0}, H^{+}$and $A^{0}$ masses is relaxed. From Eqs. (8) and (9) it follows that their contribution is positive, provided $M_{H^{+}} \gtrsim$ $M_{A}$. Obviously, the $M_{H^{+}} M_{A}$ mass splitting must increase with increasing $M_{H}$ in order to compensate for the increasingly negative $H^{0}$ contribution to $\Delta \rho_{S M}$. It is also clear that the possibility to adjust the total $\Delta \rho$ to a proper value should hold also for larger $M_{h}$ and/or $\sin ^{2}(\beta-\alpha) \neq 0$. We have checked that, for example for $M_{h}=50 \mathrm{GeV}$ and $\sin ^{2}(\beta-\alpha)=0.2$ (see Fig. 1), the plots look very similar to those for $M_{h}=20 \mathrm{GeV}$ and $\sin ^{2}(\beta-\alpha)=0$.

Since the contribution of $H^{+}, A^{0}$ and $h^{0}$ to $\Delta \rho$ depends on differences of the masses squared (quadratic violation of the $S U_{V}(2)$ "custodial" symmetry), the cancellation between their contribution and $\Delta \rho_{S M}$ which is needed in the case of $M_{h} \lesssim 20 \mathrm{GeV}$ to adjust $\Delta \rho$ to a proper value, becomes more and more delicate as the mass of $M_{H^{+}}$increases. This leads to a stronger and stronger correlation of $M_{H^{+}}$with $M_{A}$, clearly seen in Figs. 5 (note the different mass scales in the panels). This correlation becomes particularly fine-tuned in the case of small tan $\beta$, where the requirement of good $R_{b}$ forces $M_{H^{+}}$to be large. The lower limits imposed on $M_{H^{+}}$by $b \rightarrow s \gamma$ and $R_{b}$ are shown in Fig. 6 a by the solid and dashed lines, respectively. Since the very strong correlation of $M_{H^{+}}$with $M_{A}$ may seem unnatural, it is interesting to see how the requirement of "naturalness" of the $\chi^{2}$ constrains the parameter space. This is illustrated by the dotted (dash-dotted) line in Fig. 6a, which bounds from below the region in the $\left(\tan \beta, M_{H^{+}}\right)$ 
plane in which a change by $\lesssim 1 \%(3 \%)$ of $M_{A}$ value which gives the minimum of $\chi^{2}$ (for that particular point in the $\left(\tan \beta, M_{H^{+}}\right)$plane), does not lead to $\chi^{2}>19.5$. In producing these curves, the fit was always optimized with respect to the values of $M_{H}$ and $m_{t} .{ }^{4}$ It is also interesting to note (see Figs. 5) that in the case of heavy $H^{+}$, only relatively light $H^{0}$ can givea a good $\chi^{2}$ fit to the data. Therefore, for fixed values of $M_{H^{+}}$and $\tan \beta$ the requirement of $\chi^{2}<19.5$ leads to an upper bound on $M_{H}$ shown in Fig. $6 \mathrm{~b}$ (where we have optimized the fit with respect to $M_{A}$ and $m_{t}$ ).

Larger value of $\chi^{2}$ at the minimum for heavier $H^{0}$ observed in Figs. 5 can be explained by the behaviour of the parameter $S$ [31], which was not taken into account in the above discussion. We define $S$ at $q^{2}=M_{Z}^{2}$ rather than at $q^{2}=0$ (since it is $S\left(M_{Z}^{2}\right)$ that parametrizes more effectively the electroweak observables measured at LEP):

$$
S=\frac{4 s_{W}^{2}}{\alpha}\left[c_{W}^{2} F_{Z Z}\left(M_{Z}^{2}\right)-c_{W}^{2} F_{\gamma \gamma}\left(M_{Z}^{2}\right)+\frac{c_{W}}{s_{W}}\left(2 s_{W}^{2}-1\right) F_{Z \gamma}\left(M_{Z}^{2}\right)\right],
$$

where

$$
F_{i j}\left(q^{2}\right) \equiv \frac{\Pi_{i j}\left(q^{2}\right)-\Pi_{i j}(0)}{q^{2}} .
$$

For $q^{2}=0$ it is easy to obtain the analytic expression for $S(0)$, which we record in the Appendix. Since for approximately fixed $M_{A}$ the parameter $S$ grows with increasing $M_{H}$, as shown in Fig. 7 , it is obvious that for too high values of $M_{H}$ the quality of the fit will be spoiled.

When $M_{H^{+}}$increases, its negative contribution decreases $S$, but this decrease is almost entirely compensated by the change in $M_{A}$, which is required by the $\Delta \rho$ variable so that $\chi^{2}$ remains in its minimum (for that value of $M_{H^{+}}$). Therefore, the upper limit on $M_{H}$ is, for $\tan \beta \gtrsim 1.5$, almost independent of the assumed value of $M_{H^{+}}$as seen in Fig. 6b. For smaller $\tan \beta$, however, a lighter $H^{+}$induces a larger negative contribution to $\delta R_{b}$, which has the effect that the upper limit on $M_{H}$ is stronger for lighter $H^{+}$, since the minimum of $\chi^{2}$ is already higher than for heavier $H^{+}$. Therefore, this limit is rather stringent for $0.5 \lesssim \tan \beta \lesssim 0.8$.

In the case of light $h^{0}$ and $\tan \beta \gtrsim 20$, a qualitatively new behaviour of $\chi^{2}$ appears as a result of the interplay of $h^{0}, A^{0}$ and $H^{+}$contributions to $R_{b}$ (for $\sin ^{2}(\beta-\alpha) \approx 0$ the $H^{0}$ boson contributes negligibly to $R_{b}$ ). Because of the tight correlation of $M_{A}$ with $M_{H^{+}}$(required by $\Delta \rho$ ), no acceptable $\chi^{2}$ can be obtained for $H^{+}$too heavy since there is then a large negative contribution to $R_{b}$ due to the large mass splitting between $h^{0}$ and $A^{0}$. Thus, for large $\tan \beta$, very light $h^{0}$ necessarily implies the existence of relatively light $H^{+}$. The corresponding pattern of $\chi^{2}$ (for fixed $m_{t}=174 \mathrm{GeV}$ ) is illustrated in Fig. 8 for two different values of $M_{H^{+}}$and $\tan \beta=20$, 35 and 50. Deeper minima of $\chi^{2}$ for larger values of $M_{H}$ seen in Figs. 8c-f can be explained by the fact that (for fixed $M_{H^{+}}$) larger $M_{H}$ requires lighter $A^{0}$ to give acceptable $\Delta \rho$ (the correlation seen already in Fig. 5) and this increases the positive contribution to $R_{b}$ of the latter. From the pattern seen in Fig. 8, it follows that there exists, for a given mass of the

\footnotetext{
${ }^{4}$ Whenever we optimize with respect to $m_{t}$, the top mass measurement $m_{t}=(173.9 \pm 5) \mathrm{GeV}[18]$ is included as one of the fitted data in our $\chi^{2}$ fit.
} 

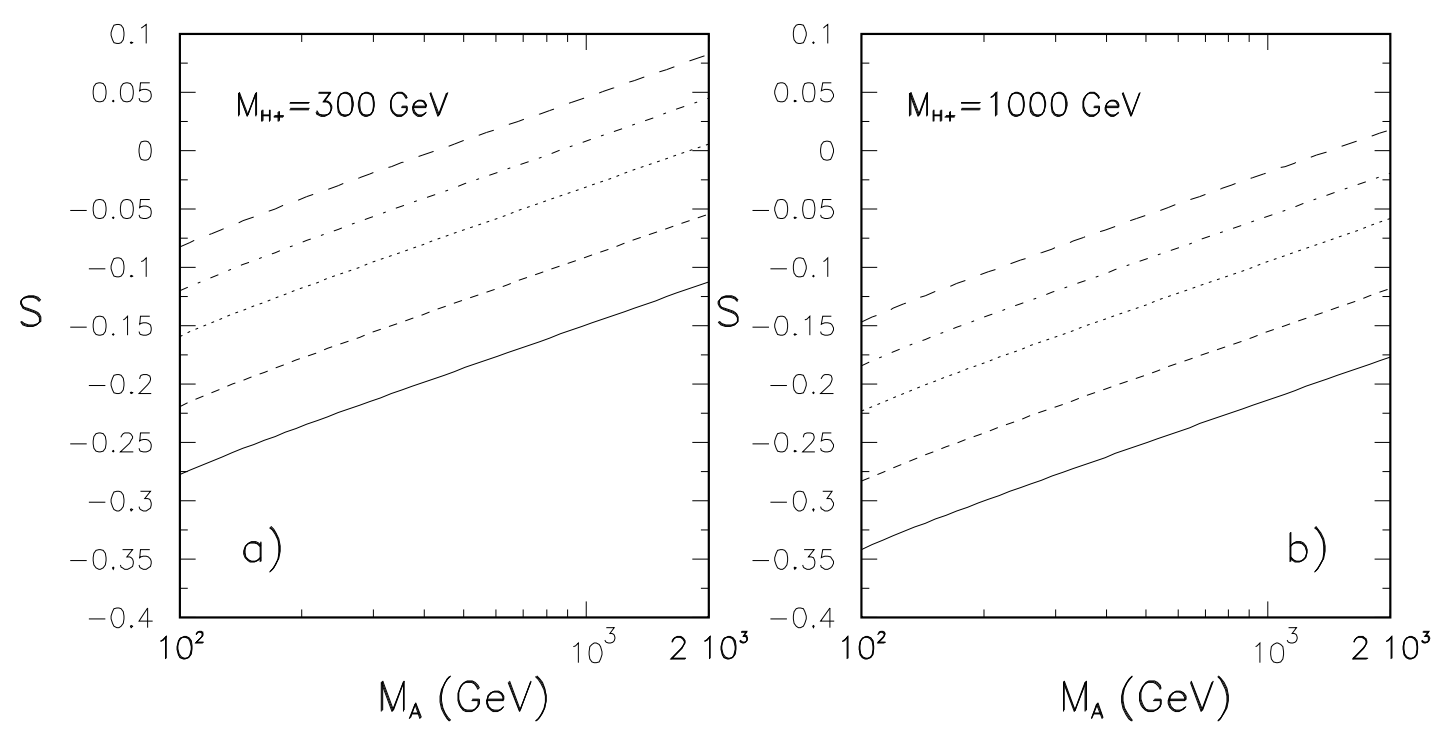

Figure 7: Parameters $S$ as a function of $M_{A}$ for $M_{H^{+}}=300 \mathrm{GeV}$ and $1 \mathrm{TeV}$ for $M_{H}=$ (from below) 100, 250, 500, 1000 and $2000 \mathrm{GeV}$. In all cases $M_{h}=10 \mathrm{GeV}$ and $\sin ^{2}(\beta-\alpha)=0$.

lighter scalar $h^{0}$ and for a given upper bound on $M_{H}$, an upper bound on $M_{H^{+}}$. For $M_{h}=20$ and $10 \mathrm{GeV}$, this bound (obtained for $\sin (\beta-\alpha)=0$ by scanning over $M_{H}, M_{A}$ and $m_{t}$ and looking for points with $\left.\chi^{2}<19.5\right)$ is plotted in Fig. 9a, for an assumed upper limit on $M_{H}$ equal to 1000,3000 and $5000 \mathrm{GeV}$. Taking $h^{0}$ lighter strengthens the bound, as can be easily inferred from Fig. 4. Of course, for large $\tan \beta$ values allowing for heavier $H^{0}$ weakens the upper bound on $M_{H^{+}}$. However, for $\tan \beta$ values $\sim 20-30$, where $R_{b}$ gradually ceases to be so important (even allowing $M_{H}$ to vary up to $3 \mathrm{TeV}$ ), the minimum of $\chi^{2}$ (for given $M_{H^{+}}$) is achieved for $M_{H}=90 \mathrm{GeV}$ (lower limit on the SM-like $M_{H}$ from direct searches). Therefore the minimum of $\chi^{2}$ does not depend on the assumed upper limit on $M_{H}$. The importance of this upper bound on $M_{H}$ taken together with the lower one coming from $b \rightarrow s \gamma$ is obvious ${ }^{5}$.

Since the best $\chi^{2}$ is obtained, for $\tan \beta \gtrsim 25$, for the lowest possible mass of the charged Higgs boson and $M_{H}$ equal to its assumed upper bound, for a given value of $\tan \beta$ there exists a lower bound on $M_{h}$ that follows from the assumed upper bound on $M_{H}$ and the lower limit on $M_{H^{+}}$coming from $b \rightarrow s \gamma$. This bound (obtained by scanning over $m_{t}$ and $M_{A}$ as well as over $\sin ^{2}(\beta-\alpha)$ in the experimentally allowed range shown in Fig. 1) is shown in Fig. 9b for two different lower bounds on $M_{H^{+}}$taken to be $200 \mathrm{GeV}$ (our limit from $b \rightarrow s \gamma$ ) and $250 \mathrm{GeV}$ and different upper bounds on $M_{H}$. (For $M_{H^{+}}=200 \mathrm{GeV}$, the upper limit on $M_{h}$ does not decrease when larger $M_{H}$ are allowed, since it would require $M_{A}<65 \mathrm{GeV}$, which is excluded by the

\footnotetext{
${ }^{5}$ However, one should note that, for given values of the Higgs sector parameters, the minimum of $\chi^{2}$ for $\tan \beta$ close to 50 occurs for $m_{t} \approx 166 \mathrm{GeV}$, for which the lower bound on $M_{H^{+}}$coming from $b \rightarrow s \gamma$ is slightly (by about $20 \mathrm{GeV}$ ) lower than the bound plotted in Fig. 6a (which was obtained for $m_{t}=174 \mathrm{GeV}$ )
} 

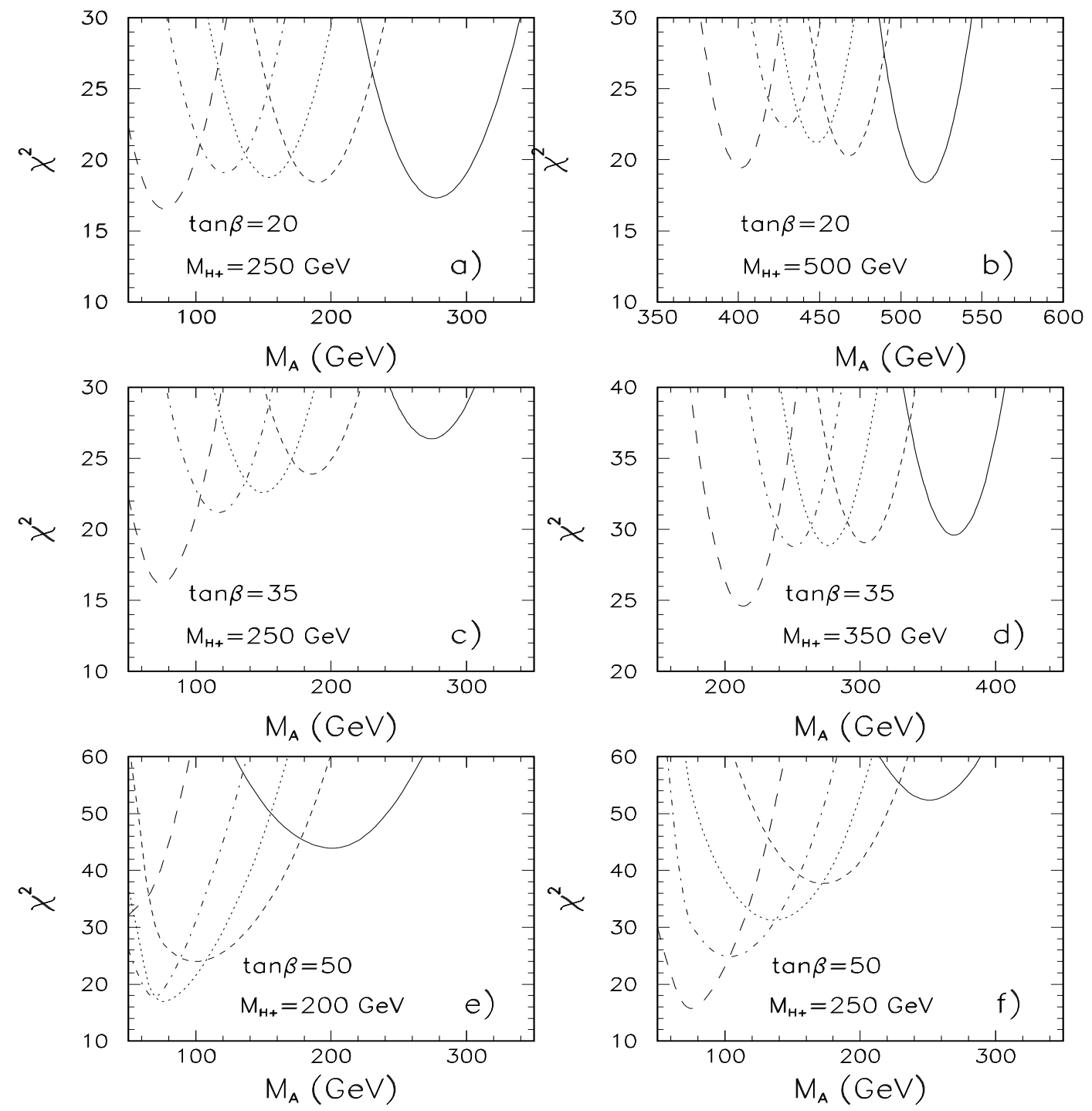

Figure 8: $\chi^{2}$ as a function of $M_{A}$ for three different values of $\tan \beta$ and different $H^{+}$masses for $M_{h}=20 \mathrm{GeV}$ (i.e. $\sin ^{2}(\beta-\alpha)=0$ ). Solid, dashed, dotted, dot-dashed and long-dashed lines correspond to $M_{H}=90,500,1000,2000$ and $5000 \mathrm{GeV}$, respectively. 

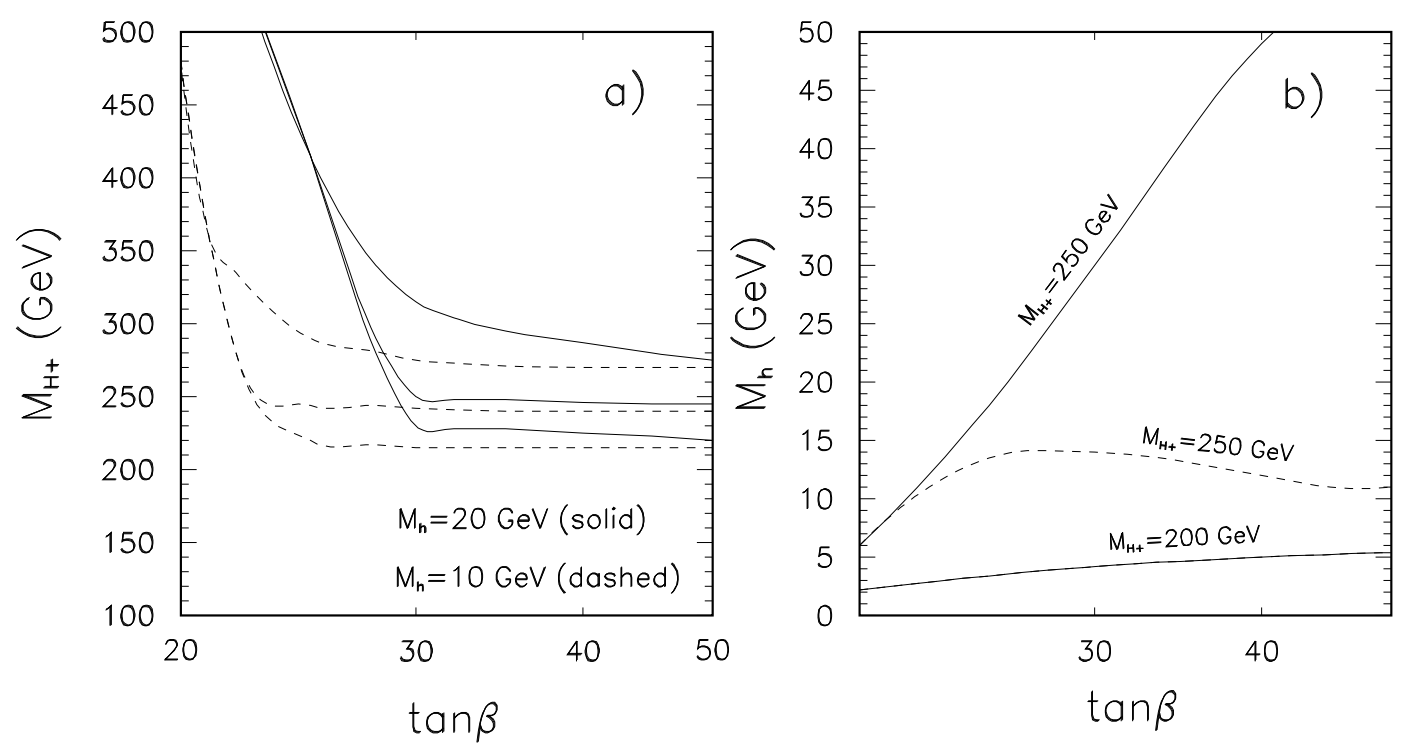

Figure 9: Limits from the $\chi^{2}$ fit: a) Upper for the $H^{+}$mass as a function of $\tan \beta$ for $M_{h}=20$ (solid lines) and $10 \mathrm{GeV}$ (dashed lines) assuming the upper limit on $M_{H}$ equal (from bottom to top) to 1,3 and $5 \mathrm{TeV}$. b) Lower for $M_{h}$ for $M_{H^{+}}=200$ and $250 \mathrm{GeV}$ (solid lines) assuming the upper limit on $M_{H}$ equal to $1 \mathrm{TeV}$, and for $M_{H^{+}}=250 \mathrm{GeV}$ (dashed line) and $M_{H}<3$ $\mathrm{TeV}$.

OPAL analysis, see Fig. 2.) The bound turns out to be very sensitive to the lower limit on $M_{H^{+}}$, showing that the $b \rightarrow s \gamma$ process is crucial for constraining a light scalar Higgs scenario for large $\tan \beta$.

The global limits shown in Figs. 9a,b must be confronted with the recent analysis [16] of the Yukawa process for $h^{0}$ production. In the case of Fig. 9a parts of the solid (dashed) lines corresponding to $\tan \beta \gtrsim 40$ (30) seem to be excluded by the data for the Yukawa process. However, those parts of these lines that are not excluded still provide interesting and complementary limits on the light $h^{0}$ scenario. In the case of Fig. $9 \mathrm{~b}$ the limit obtained for $M_{H^{+}}=200 \mathrm{GeV}$ is for most of the $\tan \beta$ range weaker than the limit imposed by the Yukawa process. For heavier $H^{+}$these limits shown in Fig. 9b become competitive to the ones derived in ref. [16].

\subsection{Light $A^{0}$}

In the the case of light $A^{0}$ the analysis is more involved because there is effectively one more variable: $\sin ^{2}(\beta-\alpha)$. Therefore let us begin again with the case of triple degeneracy, $M_{h}=$ $M_{H}=M_{H^{+}}$. As is clear from Eq. (8) the contribution of the Higgs sector to $\Delta \rho$ is in this limit independent of the value of $M_{A}$ and of $\sin ^{2}(\beta-\alpha)$; it is given simply by $\Delta \rho_{S M}\left(M_{h}\right)$. Therefore, 
for $M_{h}=M_{H}=M_{H^{+}}$and moderate values of $\tan \beta(1.5 \lesssim \tan \beta \lesssim 20)$, the best value of $\chi^{2}$ depends on the lower limit on $M_{H^{+}}$from $b \rightarrow s \gamma$. For $\tan \beta \gtrsim 1$ the condition $\chi^{2}<19.5$ is satisfied if $M_{H^{+}}<240 \mathrm{GeV}$, which is still allowed (see Fig. 6). For $\tan \beta \lesssim 1$ the light $H^{+}$that is needed to give good $\Delta \rho$ tends to give too negative a contribution to $R_{b}$ (see Fig. 6a) and the condition $\chi^{2}<19.5$ cannot be satisfied.

Even for $M_{H^{+}}$larger than $240 \mathrm{GeV}$, the value of $\chi^{2}$ can be kept below 19.5 by the departure from the limit of triple degeneracy. For example, consider the limit in which $h^{0}$ and $H^{0}$ remain degenerate (which makes $\Delta \rho$ independent of $\sin ^{2}(\beta-\alpha)$ ). It is then easy to see that for any value of $M_{H^{+}}$(and any $M_{A}$ ) there exists a solution to the equation $\Delta \rho=0$ that occurs for $M_{h}=M_{H} \lesssim M_{H^{+}}$. For values of $\tan \beta \lesssim 20$ (for which neutral Higgs bosons do not play any role in $R_{b}$ ) and $M_{H^{+}}$not too large (so that the effects of the $S$ parameter are not too large, see below) the existence of such a solution is sufficient to ensure small values of $\chi^{2}$. Note, however, that since the solution to the equation $\Delta \rho=0$ is due to the cancellation of the $\Delta \rho_{N E W}$, which depends on the mass splittings quadratically against $\Delta \rho_{S M}\left(M_{h}\right)$ which depends on $M_{h}$ only logarithmically, such a solution is strongly fine-tuned (the more, the heavier $\mathrm{H}^{+}$). The finetuning can be, however, reduced (or, more precisely, shifted to the variable $\sin ^{2}(\beta-\alpha)$ ) by relaxing the condition $M_{h}=M_{H}$. This is illustrated in Fig. 10 where, for $M_{A}=10 \mathrm{GeV}$ and $m_{t}=174 \mathrm{GeV}$, we show $\chi^{2}$ as a function of $M_{H}$ for different (moderate and low) values of $\tan \beta$ and different choices of $M_{H^{+}}$and $M_{h}$. In all cases we optimize $\chi^{2}$ with respect to the value of $\sin ^{2}(\beta-\alpha)$.

The peculiar dependence of $\chi^{2}$ as a function of $M_{H}$ seen in Fig. 10 can be understood by inspection of the formulae for $\Delta \rho$, Eq. (8). Consider first the case of $M_{A}=10 \mathrm{GeV}, M_{H^{+}}=300$ $\mathrm{GeV}$, as in Fig. 10a. For $M_{h}=90 \mathrm{GeV}$ and $M_{h} \lesssim M_{H} \ll M_{H^{+}}$, both factors, $\Delta_{c}$ and $\Delta_{s}$, are positive and obviously cannot cancel the positive first term in Eq. (8) for any choice of $\sin ^{2}(\beta-\alpha)$. As $M_{H}$ increases, however, $\Delta_{s}$ decreases rather fast and takes on negative values for $M_{H} \lesssim M_{H^{+}}$, whilst $\Delta_{c}$ decreases slowly (logarithmically) and reaches negative values only for very large values of $M_{H}$. Obviously, for $M_{H}$ such that $\Delta_{s}<-\alpha A\left(M_{A}, M_{H^{+}}\right) / 4 \pi s_{W}^{2} M_{W}^{2}<\Delta_{c}$ there always exists a choice of $\sin ^{2}(\beta-\alpha)$ for which $\Delta \rho=0$. This explains the plateau in $\chi^{2}$ for light $h^{0}$ and $M_{H}>M_{H^{+}}$. ${ }^{6}$ With $M_{h}$ increasing, $\Delta_{c}$ decreases very fast. In addition, $\Delta_{s}$ decreases too, though only logarithmically. As a result, for $M_{h}$ larger than some critical value, both $\Delta_{c}$ and $\Delta_{s}$ become negative and smaller than $-\alpha A\left(M_{A}, M_{H^{+}}\right) / 4 \pi s_{W}^{2} M_{W}^{2}$, and again $\Delta \rho$ cannot vanish leading to large values of $\chi^{2}$ seen in Figs. 10a-c for mass configurations corresponding to dot-dashed and long-dashed lines. It should also be obvious that for very heavy $H^{+}$the fine-tuning in $\sin ^{2}(\beta-\alpha)$ becomes extremely big, making such solutions rather unnatural. Thus light $A^{0}$ and values of $\tan \beta \lesssim 1$, for which $M_{H^{+}}$must be large, are rather unlikely.

For very large $M_{H^{+}}, \Delta \rho$ can vanish only for heavy $H^{0}$. In the case of heavy $h^{0}$ this means that the parameter $S$ is also large (see Fig. 7) and spoils the quality of the fit. This explains why, in Fig. 10c, for larger values of $M_{h}$, and even for the other masses in configurations for which $\Delta \rho$ can vanish by a judicious adjustment of $\sin ^{2}(\beta-\alpha)$, the value of $\chi^{2}$ is still above

\footnotetext{
${ }^{6}$ Eventually, for $M_{H}$ large enough, also $\Delta_{c}$ becomes smaller than $-\alpha A\left(M_{A}, M_{H^{+}}\right) / 4 \pi s_{W}^{2} M_{W}^{2}$, so that there is again no solution with $\Delta \rho=0$. This happens for smaller $M_{H}$, the smaller the value of $M_{H^{+}}$.
} 

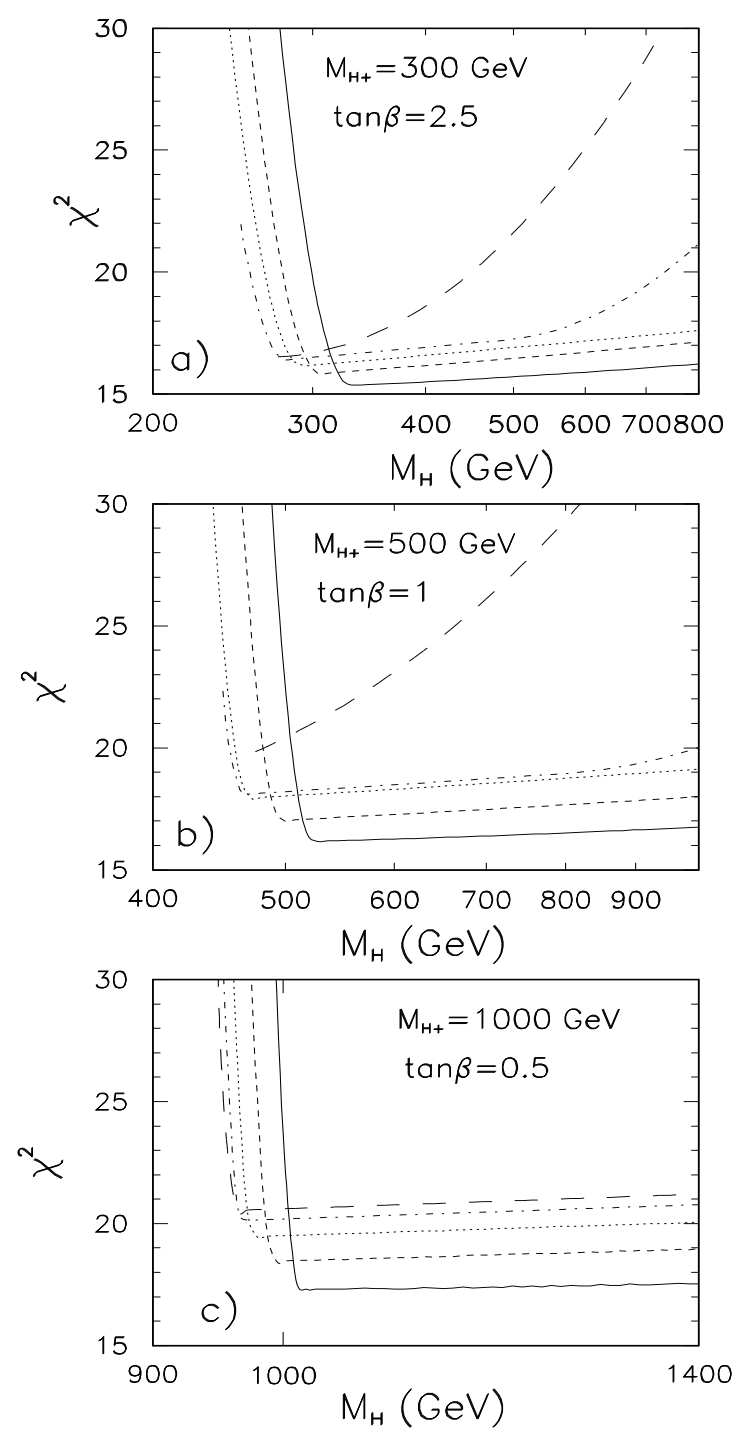

Figure 10: $\chi^{2}$ as a function of $M_{H}$ for different low and intermediate values of $\tan \beta$ and different $H^{+}$masses, for $M_{A}=10 \mathrm{GeV}$. Solid, dashed, dotted, dot-dashed and long-dashed lines correspond to $M_{h}$ equal $(90,150,200,250,275) \mathrm{GeV},(90,200,400,450,475) \mathrm{GeV}$ and (90, 250, 500, 750, 940) GeV for panels a, b and c, respectively. 
19.5.

From the above explanation it is clear that for small and intermediate values of $\tan \beta$ a light $C P$-odd scalar $A^{0}$ can be tolerated provided $h^{0}$ is lighter than some bound $M_{B}$, which is only slightly smaller than the mass of the charged Higgs boson (which in turn is constrained by $b \rightarrow s \gamma$ and, for $\tan \beta<1$, by the $R_{b}$ measurements). At the same time, $M_{H}$ is bounded from below also by roughly the same mass $M_{B}$. Of course, according to our previous discussion, the case $M_{h}=M_{H} \lesssim M_{H^{+}}$is always allowed (in this case $\sin ^{2}(\beta-\alpha)$ is completely unconstrained). It is also worth noting that, for $M_{h} \ll M_{H} \approx M_{B} \approx M_{H^{+}}$, the value of $\sin ^{2}(\beta-\alpha)$ (which is crucial for the $h^{0}$ and $A^{0}$ production processes) that is needed to keep $\chi^{2}$ below 19.5 is close to 1 , implying that $M_{h}$ is constrained, in this case, by the LEP search to be greater than $\approx 90$ $\mathrm{GeV}$. For heavier $H^{0}, \sin ^{2}(\beta-\alpha)$ decreases, which means that the experimental lower bound on $M_{h}$ is also relaxed appropriately (recall that the limits from the associated $h^{0} A^{0}$ production require only $M_{h} \gtrsim 50(70) \mathrm{GeV}$ for $M_{A}=10(50) \mathrm{GeV}$ - see Fig. 2 - irrespectively of the value of $\left.\sin ^{2}(\beta-\alpha)\right)$. The decrease of $\sin ^{2}(\beta-\alpha)$ with $M_{H}>M_{H^{+}}$is slightly faster for heavier $A^{0}$ (and/or $h^{0}$ ) and slower for heavier $H^{+}$.

For large $\tan \beta$ the dependence of $\chi^{2}$ on $M_{H}$ for different values of $M_{h}$ and $M_{H^{+}}$is shown in Fig. 11. The mass of the $C P$-odd scalar is taken to be $25 \mathrm{GeV}$ so as to respect the bound from the Yukawa process also for $\tan \beta=50[15,16]$. The behaviour of $\chi^{2}$ can be explained by combining the information contained in Figs. 10 (reflecting mainly the behaviour of $\Delta \rho$ ) with the behaviour of the corrections to $R_{b}$ for different Higgs boson mass configurations. In order for corrections to the latter quantity not to be too negative the neutral scalar that couples more strongly to the $b \bar{b}$ pair cannot be too heavy. On the other hand, for $M_{H} \lesssim M_{H^{+}}$, a good $\Delta \rho$ is obtained for $\sin ^{2}(\beta-\alpha) \approx 1$ (i.e. $\sin \alpha \approx 0$ ), which means that it is the mass splitting between $H^{0}$ and $A^{0}$ that is relevant to $R_{b}$. Only for sufficiently heavy $H^{0}$ does $\sin ^{2}(\beta-\alpha)$ become small enough $(\sin \alpha \approx 1)$ so that $h^{0}$ couples with full strength to $b \bar{b}$, and becomes relevant to $R_{b}$. Thus, a good fit to the data can be obtained only with light $h^{0}$ and rather heavy $H^{0}$ (since this occurs for $\sin ^{2}(\beta-\alpha) \ll 1, h^{0}$ can be lighter than $90 \mathrm{GeV}$ as we have just explained). The increase of $\chi^{2}$ for $M_{H}>1 \mathrm{TeV}$ seen in Fig. $11 \mathrm{a}$ and $\mathrm{d}$ for $M_{h}=70$ and $90 \mathrm{GeV}$ is due to the fact that, for lighter $H^{+}, \Delta_{c}$ becomes smaller than $-\alpha A\left(M_{H^{+}}, M_{A}\right) / 4 \pi s_{W}^{2} M_{W}^{2}$ already for relatively light $H^{0}$. Since, as explained above, $\Delta_{c}$ decreases very fast as $M_{h}$ increases, this effect is even more pronounced for $M_{h}=150 \mathrm{GeV}$. Note that because of this effect for $M_{H^{+}}=200$ $\mathrm{GeV}$ one can reach $\chi^{2}<19.5$ only for $M_{h}<90 \mathrm{GeV}$.

For the same value of $M_{H}$ and $M_{h}$ the $\chi^{2}$ is slightly larger for heavier $H^{+}$(despite the fact that the negative contribution of the latter Higgs boson to $R_{b}$ is decreased), because of the behaviour of $\sin ^{2}(\beta-\alpha)$ : it is larger for heavier $H^{+}$and therefore, light $h^{0}$ does not fully compensate for the effects of light $A^{0}$ as $h^{0}$ couples more weakly to the $b \bar{b}$ pair. The conclusion following from the above considerations and from Figs. 11 is that, in the scenario with large $\tan \beta$ and light $C P$-odd neutral Higgs particle $\left(M_{A} \gtrsim 25 \mathrm{GeV}\right)$, the mass of the lighter neutral $C P$-even boson $h^{0}$ is bounded from above. This upper bounds on $M_{h}$ for four different values of the light $C P$-odd scalar mass (obtained by scanning over $m_{t}, \sin (\beta-\alpha), M_{H}$ and $M_{H^{+}}$) are shown as functions of $\tan \beta$ in Fig. 12. Of course, as $\tan \beta$ decreases, the upper limit on $M_{h}$ approaches the bound $M_{B} \lesssim M_{H^{+}}$discussed previously. The existence of an upper bound 

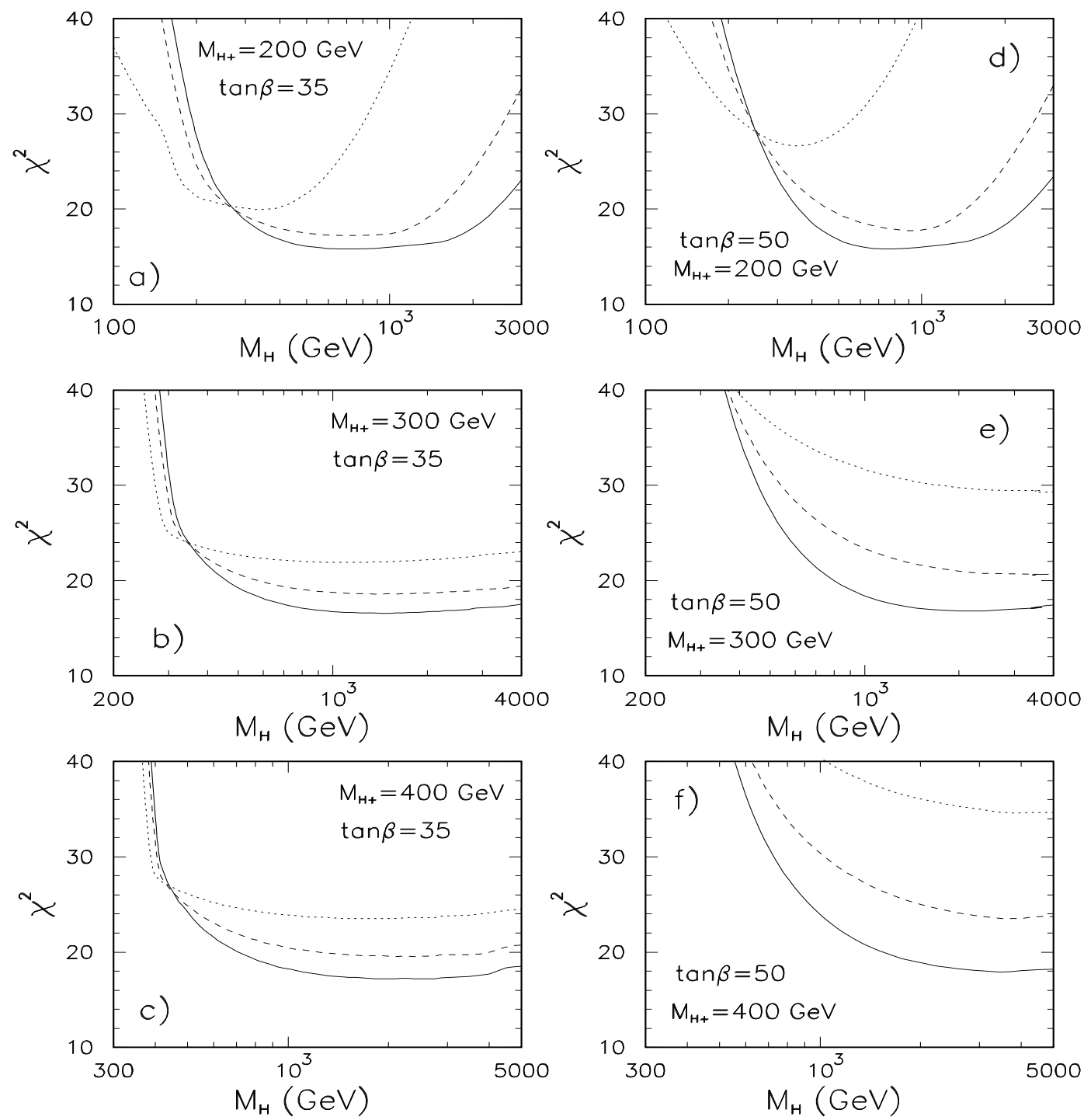

Figure 11: $\chi^{2}$ as a function of $M_{H}$ for $\tan \beta=35$ and 50 and different $H^{+}$masses. Solid, dashed, dotted lines correspond to $M_{h}$ equal to 70,90 and 150 , respectively. $M_{A}=25 \mathrm{GeV}$. 


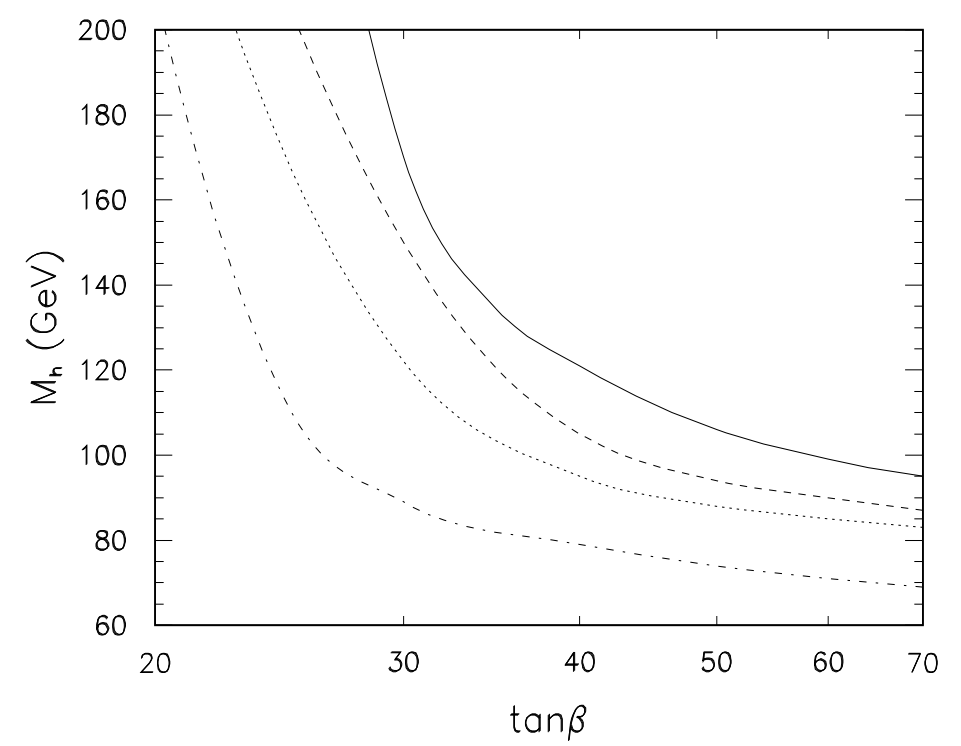

Figure 12: Upper limit on $M_{h}$ in the light $C P$-odd scalar scenario as a function of $\tan \beta$ for $M_{A}=25 \mathrm{GeV}$ (solid line), $15 \mathrm{GeV}$ (dashed line), $10 \mathrm{GeV}$ (dotted line) and $1 \mathrm{GeV}$ (dot-dashed line).

on $M_{h}$ means that LEP2 will be able to effectively test the scenario with light $A^{0}$ and large $\tan \beta$ either via the Bjorken process or the associated Higgs boson production, at least for $\tan \beta>45(30)$ for $M_{A}<25$ (10) GeV.

\section{Summary of the results of the global fit and other constraints}

We have investigated the impact of the precision electroweak data on the parameter space of the 2HDM of type II. We have been particularly interested in constraints imposed on the very light neutral scalar scenarios (with $h^{0}$ or $A^{0}$ in the range $\lesssim 25-30 \mathrm{GeV}$ ) by the requirement of good (as good as in the SM) fit to the electroweak data. It turns out that neither scenario is excluded (directly or indirectly), provided some constraints are respected.

Apart from the well-known constraints on the charged Higgs boson mass set in 2HDM(II) by $b \rightarrow s \gamma$ and, for $\tan \beta<1$, by $R_{b}$, we find that in the case of a light $C P$-even scalar $h^{0}$ and of intermediate values of $\tan \beta$ masses of the $C P$-odd and charged Higgs bosons must be tightly correlated (the more, the heavier is $\mathrm{H}^{+}$) in order to maintain in the $2 \mathrm{HDM}$ (II) the same quality of the $\chi^{2}$ fit to the data as in the SM; this leads to strong "fine-tuning" of this scenario. Therefore, limiting the acceptable degree of "fine-tuning" yields an upper bound on 
$M_{H^{+}}$(Fig. 6a). For a given mass of $H^{+}$the requirement of a good fit to the data puts also an upper limit on $M_{H}$, which is particularly strong for $\tan \beta<1$ (Fig. 6b). For large values of $\tan \beta(\tan \beta \gtrsim 20-30)$ the interplay between the corrections to $\Delta \rho$ and $R_{b}$ implies that if $h^{0}$ is light, $H^{+}$must also be light. The upper limit (as a function of $\tan \beta$ ) depends on the assumed upper bound on $M_{H}$ but, for $M_{H}$ in the TeV range, it is very strong for $\tan \beta \gtrsim 35$, and $M_{H^{+}}$ of the order of $300 \mathrm{GeV}$ (Fig. 9a). It is also interesting that, for large $\tan \beta$ and $M_{H^{+}}>200$ $\mathrm{GeV}$, the electroweak data set the lower limit on the mass of $h^{0}$ shown in Fig. 9b.

For the light $A^{0}$ scenario we find that, for low and intermediate values of $\tan \beta$, the quality of the fit is maintained provided $M_{h}<M_{B}<M_{H}$, where $M_{B} \lesssim M_{H^{+}}$. For $\tan \beta>30-35$ there emerges an additional upper bound on the mass of the lighter scalar $h^{0}$, which enables the test of the light $A^{0}$-large $\tan \beta$ configuration at LEP2.

Other potential sources of further constraints on the $2 \mathrm{HDM}(\mathrm{II})$ that we have not considered here are the muon $g-2$ measurement [34] and $Z \rightarrow h(A) \gamma$ decays [35].

The upper limit on $B R\left(Z^{0} \rightarrow h^{0}\left(A^{0}\right) \gamma\right)$ decays set by LEP1 constrain our model only for $h^{0}\left(A^{0}\right)$ masses below $20 \mathrm{GeV}$ and for values of $\tan \beta$ either very low (below 0.2) or very large, above 55-70 GeV (which we have not considered). For larger $h^{0}\left(A^{0}\right)$ masses, $\tan \beta$ is pushed outside the range (6) in which perturbative calculations can be done reliably. In this case, the resulting constraints can eventually be given a meaning as constraining the effective (on-shell) $Z^{0} h^{0} \gamma$ and $Z^{0} A^{0} \gamma$ couplings but the relation of these couplings to the original parameters of the model (which we have been using) cannot be calculated perturbatively.

The constraints following from the present measurement of the $g-2$ of the muon are stronger than the ones following from the Yukawa process only for $h^{0}\left(A^{0}\right)$ masses below $1-2 \mathrm{GeV}$, in which case they exclude values of $\tan \beta \gtrsim 4(10)$ for $M_{h(A)}=0.1$ (1) GeV. The E821 experiment may (depending on the measured central value) improve these limits (according to the analysis presented in [34]), so that they become stronger than the Yukawa process ones up to $M_{h(A)} \sim 10$ $\mathrm{GeV}$ and exclude values of $\tan \beta \gtrsim 2(15)$ for $M_{h(A)}=1(10) \mathrm{GeV}$.

\section{Appendix}

Here we give the expression for the parameter $S(0)$. It differs slightly from the formula presented in ref. [36]:

$$
\begin{array}{r}
S=-\frac{1}{6 \pi} \log \frac{M_{H^{+}}}{M_{W}}+\frac{1}{\pi} \sin ^{2}(\beta-\alpha)\left[A^{\prime}\left(M_{A}, M_{H}\right)+A^{\prime}\left(M_{W}, M_{h}\right)+M_{Z}^{2} B^{\prime}\left(M_{Z}, M_{h}\right)\right. \\
+\frac{1}{\pi} \cos ^{2}(\beta-\alpha)\left[A^{\prime}\left(M_{A}, M_{h}\right)+A^{\prime}\left(M_{W}, M_{H}\right)+M_{Z}^{2} B^{\prime}\left(M_{Z}, M_{H}\right)\right] .
\end{array}
$$

The functions $A^{\prime}$ and $B^{\prime}$ are given by:

$$
A^{\prime}\left(m_{1}, m_{2}\right)=\frac{1}{12}\left[-\frac{11}{6}+\frac{m_{1}^{2}}{m_{1}^{2}-m_{2}^{2}} \log \frac{m_{1}^{2}}{M_{W}^{2}}+\frac{m_{2}^{2}}{m_{2}^{2}-m_{1}^{2}} \log \frac{m_{2}^{2}}{M_{W}^{2}}\right.
$$




$$
\begin{gathered}
\left.+\frac{m_{1}^{4}+m_{2}^{4}}{\left(m_{1}^{2}-m_{2}^{2}\right)^{2}}-\frac{m_{1}^{2} m_{2}^{2}\left(m_{1}^{2}+m_{2}^{2}\right)}{\left(m_{1}^{2}-m_{2}^{2}\right)^{3}} \log \frac{m_{1}^{2}}{m_{2}^{2}}\right], \\
B^{\prime}\left(m_{1}, m_{2}\right)=-\frac{1}{2} \frac{m_{1}^{2}+m_{2}^{2}}{\left(m_{1}^{2}-m_{2}^{2}\right)^{2}}+\frac{m_{1}^{2} m_{2}^{2}}{\left(m_{1}^{2}-m_{2}^{2}\right)^{3}} \log \frac{m_{1}^{2}}{m_{2}^{2}}
\end{gathered}
$$

\section{Acknowledgments}

P.H.Ch. has been partly supported by the Polish State Committee for Scientific Research grant 2 P03B 03014 (for 1998-1999). M.K. has been partly supported by the U.S.-Polish Maria Skłodowska-Curie Joint Fund II (MEN/DOE-96-264) and by the Polish State Committee for Scientific Research grants 2 P03B 01414 and 2 P03B 18410.

We would like to thank F. Borzumati, C. Greub and M. Misiak for discussions. M.K. would also like to thank J. Gunion and W. Hollik for important suggestions, H. Haber for discussions and hospitality during her stay at the Santa Cruz University, and K. Desh for providing some experimental data.

\section{References}

[1] LEP Electroweak Working Group, CERN report LEPEWWG/98-01.

[2] M. Krawczyk, in proceedings of the XXXI Rencontres de Moriond "Electroweak Interactions and Unified Theories" Les Arcs, France, March 1996, ed. Tran Thanh Van, World Scienific, (hep-ph/9607268), Proceedings of the XXVIII Rochester Int. Conf. on High Energy Physics, Warsaw, Poland, July 1996, eds. Z. Ajduk, A.K. Wróblewski, World Scientific, p. 1460; Proceedings of the Cracow School of Theoretical Physics, Zakopane, Poland, January 1999, Acta Phys. Pol. B29 (1999) 3543.

[3] A.K. Grant, Phys. Rev. D51 (1995) 207.

[4] W.F.L. Hollik, Z. Phys. C32 (1986) 291; C37 (1988) 568.

[5] H.E. Haber and K. Logan, talk at the First European Meeting From Planck Scale to Electroweak Scale, Kazimierz, Poland, May 1998.

[6] F. Cornet, W.F.L. Hollik and W. Mösle, Nucl. Phys. B428 (1994) 61.

[7] J.F. Gunion, H.E. Haber, G.L. Kane and S. Dawson, The Higgs Hunter's Guide, AddisonWesley, 1990.

[8] H. Haber, in Perspectives on Higgs Physics II, ed. G.L. Kane, World Scientific, 1998 (hep$\mathrm{ph} / 9707213)$. 
[9] V. Berger et al., Phys. Rev. D41 (1990) 3421;

Y. Grossman, Nucl. Phys. B426 (1994) 355.

[10] ALEPH Collaboration, ALEPH 98-029 CONF 98-017;

K. Moenig for the DELPHI Collaboration, talk at the LEPC Meeting, CERN, 31 March 1998;

M. Acciarri et al. (L3 Collaboration), preprint CERN-EP/98-052;

OPAL Collaboration, OPAL Physics Note PN340, March 1998;

V. Ruhlmann-Kleider, talk at the XXX Rencontres de Moriond "QCD and Hadronic Interactions", Les Arcs, France, March 1998;

M. Felcini, talk at the XXXIV Rencontres de Moriond "Electroweak Interactions and Unified Theories", Les Arcs, France, March 1999.

[11] G. Abbiendi et al. (the OPAL Collaboration), Eur. Phys. J. C7 (1999) 407.

[12] M. Acciari et al. (L3 Collaboration), Z. Phys. C62 (1994), 551, paper PA11-016 submited to the XXVIII Rochester Int. Conf. on High Energy Physics, Warsaw, Poland, July 1996.

[13] F. Wilczek, Phys. Rev. Lett. 39 (1977) 1304.

[14] P. Lee-Franzini, in Proceedings of the XXIV Rochester Int. Conf. on High Energy Physics, Munich, Germany, 1988, eds. R. Kotthaus and J. H. Kühn, p. 1432;

M. Narain, Ph.D. Thesis, Inclusive photon spectra from $\Upsilon$, State Univ. of New York at Stony Brook, 1991;

S. T. Lowe, Ph.D.Thesis, A search for narrow states in radiative $\Upsilon$ decays, SLAC, 1986;

D. Antreasyan et al. (Crystall Ball Collaboration), Phys. Lett. B251 (1990) 204.

[15] The ALEPH Collaboration, paper PA13-027 submitted to the XXVIII Rochester Int. Conf. on High Energy Physics, Warsaw, Poland, July 1996.

[16] P. Zalewski, private communication.

[17] M. Felcini, in ref. [10];

B. Bevensee, Proceedings of the XXXIII Rencontres de Moriond "Electroweak Interactions and Unified Theories" Les Arcs, France, March 1998, ed. Tran Thanh Van, World Scienific (preprint FERMILAB-CONF-98-1555-E).

[18] M. Jones (for D0 and CDF Collaborations), talk at the XXXIII Rencontres de Moriond "Electroweak Interactions and Unified Theories", Les Arcs, France, March 1998.

[19] J. Ellis, G.L. Fogli and E. Lisi, Phys. Lett. B389 (1996) 321 and references therein; P.H. Chankowski and S. Pokorski, Phys. Lett. B356 (1995) 307 and hep-ph/9509207.

[20] P.H. Chankowski and S. Pokorski, Phys. Lett. B366 (1996) 188, and in Perspectives on Supersymmetry, ed. G.L. Kane, World Scientific (hep-ph/9707497); W. de Boer, A. Dabelstein, W.F.L. Hollik, W. Mösle and U. Schwickerath, Z. Phys. C75 (1997) 627 and preprint IEKP-KA-96-08 (hep-ph/9609209). 
[21] G. Altarelli, R. Barbieri and S. Jadach, Nucl. Phys. B396 (1992) 3;

J.L. Hewett, T. Takeuchi and S. Thomas, preprint SLAC-PUB-7088 (hep-ph/9603391) in Electroweak Symmetry Breaking and Beyond the Standard Model eds. T. Barklow, S. Dawson, H.E. Haber and S. Siegriest, World Scientific.

[22] J. Rosiek Phys. Lett. B252 (1990) 135;

A. Denner, R.J. Guth, W.F.L. Hollik and J.H. Kühn, Z. Phys. C51 (1991) 695;

M. Boulware and D. Finnell, Phys. Rev. D44 (1991) 2054.

[23] P.H. Chankowski and S. Pokorski, Nucl. Phys. B475 (1996) 3.

[24] CLEO Collaboration, paper ICHEP98 No. 1011 submitted to the XXIX Rochester Int. Conf. on High-Energy Physics, Vancouver, B.C., Canada, 1998, (preprint CLEO CONF 98-17).

[25] A.J. Buras, M. Misiak, M. Münz and S. Pokorski, Nucl. Phys. B424 (1994) 374.

[26] K. Adel and Y.P. Yao, Phys. Rev. D49 (1994) 4945;

C. Greub, T. Hurth and D. Wyler, Phys. Lett. B380 (1996) 385 and Phys. Rev. D54 (1996) 3350.

[27] M. Ciuchini, G. Degrassi, P. Gambino and G.-F. Giudice, Nucl. Phys. B527 (1998) 21;

P. Ciafaloni, A. Romanino and A. Strumia, Nucl. Phys. B524 (1998) 361.

[28] A. Czarnecki and W.A. Marciano, Phys. Rev. Lett. 81 (1998) 277; A. Kagan and M. Neubert, Eur. Phys. J. C7 (1999) 5.

[29] K. Chetyrkin, M. Misiak and M. Münz, Phys. Lett. B400 (1997) 206.

[30] C. Greub and F.M. Borzumati, talk by C.G. at the XXIX Rochester Int. Conf. on High Energy Physics, Vancouver, B.C., Canada, Jul. 1998, (hep-ph/9810240).

[31] M. Peskin and T. Takeuchi Phys. Rev. Lett. 65 (1990), 964, Phys. Rev. D46 (1992) 381.

[32] A. C. Bawa and M. Krawczyk, Contribution to the HERA Working Group, Warsaw preprint IFT 16/91 and Erratum; IFT 17/93; Phys. Lett. B 357 (1995) 637;

M. Krawczyk, Proceedings of the Workshop Future Physics at HERA, 1995-96, p. 244 (hep-ph/9609477).

[33] J. Kalinowski and M. Krawczyk, Phys. Lett. B361 (1995) 66, Acta Phys. Pol. B27 (1996) 961.

[34] M. Krawczyk and J. Żochowski, Phys. Rev. D55 (1997) 6968.

[35] M. Krawczyk, P. Mättig and J. Żochowski, Eur. Phys. J. C8 (1999) in press (hep$\mathrm{ph} / 9811256)$.

[36] T. Inami, C.S. Lim and A. Yamada, Mod. Phys. Lett. A7 (1992) 2789. 